\title{
Propagators and Renormalization Transformations for Lattice Gauge Theories. II
}

\author{
T. Bałaban* \\ Department of Physics, Harvard University, Cambridge, MA02138, USA
}

\begin{abstract}
We continue the studies of the Paper I and extend the results of this paper to operators defined by restrictions on different scales, or by renormalization transformations of different orders.
\end{abstract}

\section{Introduction}

In our study of ultra-violet stability for gauge field theories we will have to consider propagators defined by some quadratic forms with restrictions of different scales on disjoint subdomains of a lattice. In this paper we will continue the investigation of the fundamental quadratic form $\langle\partial A, \partial A\rangle$, and the restrictions are given by averaging operators of different orders, introduced in the first part [4]. We will be interested in the same properties as before, thus in local regularity properties and in exponential decay, but we will have to relate them to many scales appearing in the problem.

This paper is a continuation of [4] and we use all the notations introduced there without further explanation. We refer to the results and the formulas of this paper using its original numbering, for example Proposition 1.3, formula (1.81), and so on. The only notation we would like to mention here is that a distance between two points $x, y \in \eta Z^{d}, \eta>0$, is given by the $l^{1}$-norm of the vector $x-y$ and is denoted by $|x-y|$, i.e. $|x-y|=\sum_{\mu=1}^{d}\left|x_{\mu}-y_{\mu}\right|$. This distance depends of course on the scale of the lattice.

\section{A. Operators Defined by Conditions on Many Scales}

Let us begin with a generalization of the variational problem considered in Sect. D of [4] and leading to the operator $H_{k}$. At first we have to describe a geometry of

* $\quad$ Research supported in part by the National Science Foundation under Grant PHY-82-03669 
domains on which we will consider the problem. We consider a sequence of domains

$$
\Omega_{1} \supset \Omega_{2} \supset \ldots \supset \Omega_{k}, \quad \Omega_{j} \subset T_{\eta}, \quad j=1,2, \ldots, k,
$$

which satisfy the following conditions:

$\Omega_{j}=B^{j}\left(\Omega_{j}^{(j)}\right), \quad \Omega_{j}^{(j)} \subset T_{L^{j} \eta}^{(j)} \quad$ and it is a sum of big blocks, $\left(L^{j} \eta\right)^{-1} \operatorname{dist}\left(\Omega_{j}^{c}, \Omega_{j+1}\right)>R M, \quad M$ is a size of big blocks and $R$ is a big positive integer which will be fixed later.

Such sequences appear e.g. in the proof of the upper bound in [2]. The above sets are subsets of $T_{\eta}$, thus sets of sites of this lattice, but we will apply the same notations to sets of bonds defined in the following way. If $\Omega \subset T_{\eta}$, then we denote by $\Omega$ also the set of bounds $\bigcup_{x \in \Omega} s t(x)=\left\{\right.$ bonds $b \subset T_{\eta}$ : at least one end-point of $b$ belongs to $\Omega$ \}. Let us define

$$
\Lambda_{j}=\Omega_{j}^{(j)} \backslash \Omega_{j+1}^{(j)}, \quad j=1, \ldots, k-1, \quad \Lambda_{k}=\Omega_{k}^{(k)}, \quad \Lambda_{0}=\Omega_{1}^{c}
$$

for the sets of sites and the sets of bonds; thus we have

$$
\Omega_{1}=\bigcup_{j=1}^{k} B^{j}\left(\Lambda_{j}\right), \quad T=\bigcup_{j=0}^{k} B^{j}\left(\Lambda_{j}\right), \text { where } B^{0}\left(\Lambda_{0}\right)=\Lambda_{0} .
$$

Let us notice that we admit the case when some domains $\Omega_{j}$ are equal to $T_{\eta}$, for example $\Omega_{j}=T_{\eta}$ for $j=1,2, \ldots, l, l \leqq k$.

The variational problem can be formulated as follows. We consider the functional

$$
A \rightarrow \sum_{p} \eta^{d}|(\partial A)(p)|^{2}
$$

for $A$ fixed outside $\Omega_{1}$ and with fixed averages inside $\Omega_{1}$, more exactly

$$
A=B_{0} \quad \text { on } \Lambda_{0}, \quad Q_{j} A=B_{j} \quad \text { on } \Lambda_{j}, \quad j=1, \ldots, k .
$$

The functional and the conditions are invariant with respect to gauge transformations $\lambda: A \rightarrow A^{\lambda}=A-\partial \lambda$ such that

$$
\lambda=0 \quad \text { on } \quad \Lambda_{0}, \quad Q_{j}^{\prime} \lambda=0 \quad \text { on } \quad \Lambda_{j}, \quad j=1, \ldots, k .
$$

These gauge transformations form a group and we consider orbits of this group. We want to find a minimal orbit of the functional (2.5) under the restrictions (2.6). To solve this problem we have to fix a convenient gauge condition. We will use a generalization of the gauge condition $R \partial^{*} A=0$ defined and used in Sects. C and D of [4]. This generalization can be defined in the following way: from each orbit of the gauge group we choose a minimum of the functional

$$
\begin{aligned}
& \sum_{x} \eta^{d}\left|\left(\partial^{*} A^{\lambda}\right)(x)\right|^{2}=\sum_{x} \eta^{d}\left|\left(\partial^{*} A\right)(x)-(\Delta \lambda)(x)\right|^{2}, \\
& \lambda=0 \quad \text { on } \quad \Lambda_{0}, \quad Q_{j}^{\prime} \lambda=0 \quad \text { on } \quad \Lambda_{j}, \quad j=1, \ldots, k .
\end{aligned}
$$

If $\lambda_{0}$ defines a minimum, then we have the equation

$$
\sum_{x} \eta^{d}(\Delta \delta \lambda)(x)\left(\left(\partial^{*} A\right)(x)-\left(\Delta \lambda_{0}\right)(x)\right)=0,
$$


where $\lambda_{0}, \delta \lambda$ satisfy (2.7) and besides this $\delta \lambda$ is arbitrary. Let us denote

$$
N\left(Q^{\prime}\right)=\{\lambda: \lambda \text { satisfies }(2.7)\},
$$

and let $R$ be an orthogonal projection in the space $L^{2}\left(T_{\eta}\right)$ onto the subspace $\Delta N\left(Q^{\prime}\right)$. Equation (2.9) implies $\Delta \lambda_{0}=R \partial^{*} A$, and this equation has exactly one solution because the Laplace operator $\Delta$ is positive on the subspace $N\left(Q^{\prime}\right)$, hence it is invertible on this subspace. More exactly we have the inequality

$$
\langle\lambda, \Delta \lambda\rangle \geqq \pi^{2} \sum_{j=1}^{k}\left(L^{j} \eta\right)^{-2} \sum_{x \in B^{J}\left(\Lambda_{j}\right)} \eta^{d}|\lambda(x)|^{2}, \quad \lambda \in N\left(Q^{\prime}\right),
$$

as it follows from [3, 2.26, and 2.27]. Thus the functional (2.8) has exactly one minimum on each orbit. This miminum satisfies the equation

$$
R \partial^{*} A^{\lambda_{0}}=0, \quad \text { or } R \partial^{*} A=0 \quad \text { if we take } A^{\lambda_{0}} \text { as } A \text {. }
$$

Now we will obtain a representation of the operator $R$ similar to the representation obtained in Sect. C. According to the definition a value $R f$ of the operator $R$ acting on a function $f$ defined on $T_{\eta}$ is equal to $\Delta \lambda$, where $\lambda$ is a minimum of the functional

$$
\lambda \in N\left(Q^{\prime}\right), \quad \lambda \rightarrow \sum_{x} \eta^{d}|f(x)-(\Delta \lambda)(x)|^{2}=\sum_{x} \eta^{d}\left|f(x)-\left(\Delta_{a}^{\prime} \lambda\right)(x)\right|^{2},
$$

and where $\Delta_{a}^{\prime}=\Delta+Q^{\prime *} a Q^{\prime}$ and the operator $Q^{\prime *} a Q^{\prime}$ is given by the quadratic form

$$
\left\langle\lambda, Q^{*} a Q^{\prime} \lambda\right\rangle=\sum_{j=0}^{k} \sum_{y \in \Lambda_{j}} a_{j}\left(L^{j} \eta\right)^{d-2}\left|\left(Q_{j}^{\prime} \lambda\right)(y)\right|^{2} .
$$

The numbers $a_{j}$ satisfy the recursive equations $a_{j+1}=a a_{j} / a L^{-2}+a_{j}, a_{1}=a$ (see $[1$, 2.13 and 2.15]), and we assume that $\left(Q_{0}^{\prime} \lambda\right)(x)=\lambda(x), x \in \Lambda_{0}$. To find this minimum we consider the function

$$
g(\lambda, \omega)=\frac{1}{2} \sum_{x} \eta^{d}\left|f(x)-\left(\Delta_{a}^{\prime} \lambda\right)(x)\right|^{2}+\left\langle\omega, Q^{\prime} \lambda\right\rangle,
$$

where

$$
\left\langle\omega, Q^{\prime} \lambda\right\rangle=\sum_{j=0}^{k} \sum_{y \in \Lambda_{j}}\left(L^{j} \eta\right)^{d} \omega(y)\left(Q_{j}^{\prime} \lambda\right)(y) .
$$

Extremal points of this function are given by solutions of the equations

$$
\frac{\delta g}{\delta \lambda}=-\Delta_{a}^{\prime}\left(f-\Delta_{a}^{\prime} \lambda\right)+Q^{* *} \omega=0, \quad \frac{\delta g}{\delta \omega}=Q^{\prime} \lambda=0 .
$$

The first equation gives $\lambda=\Delta_{a}^{\prime-1} f-\Delta_{a}^{\prime-2} Q^{* *} \omega=G^{\prime} f-G^{\prime 2} Q^{*} \omega$, from the second we get $Q^{\prime} \lambda=Q^{\prime} G^{\prime} f-Q^{\prime} G^{\prime 2} Q^{*} \omega=0$, hence $\omega=\left(Q^{\prime} G^{\prime 2} Q^{\prime *}\right)^{-1} Q^{\prime} G^{\prime} f$ and $\lambda=G^{\prime} f$ $-G^{\prime 2} Q^{*}\left(Q^{\prime} G^{\prime 2} Q^{*}\right)^{-1} Q^{\prime} G^{\prime} f$. Of course $Q^{\prime} \lambda=0$, hence

$$
R f=\Delta \lambda=\Delta_{a}^{\prime} \lambda=f-G^{\prime} Q^{*}\left(Q^{\prime} G^{\prime 2} Q^{* *}\right)^{-1} Q^{\prime} G^{\prime} f .
$$

The operator $G^{\prime}=\Delta_{a}^{\prime-1}$ is a well defined, positive operator because $\Delta_{a}^{\prime}$ satisfies the inequality (2.11) for all $\lambda$, with $\min \left\{a_{j}, \pi^{2}\right\}$ instead of $\pi^{2}$ and the index $j$ running from 0 to $k$ on the right-hand side. This implies that $G^{\prime 2}$ and $Q^{\prime} G^{\prime 2} Q^{\prime *}$ are positive operators, hence $\left(Q^{\prime} G^{\prime 2} Q^{*}\right)^{-1}$ is well defined and positive also. We will investigate later properties of these operators, and the operator $R$. 
Now let us come back to the variational problem (2.5), (2.6) with the gauge fixing condition (2.12). We will prove that there exists exactly one critical configuration, which is of course, a minimum of the functional (2.5). By definition it is a value of an operator $H$ at the configuration $B$. To investigate this operator it is convenient to express it in terms of a properly chosen Green's function of some elliptic difference operator in a manner similar to the formula (1.103). To prove the above statements we apply again the Lagrange multipliers method and we consider the functional

$h(A, \lambda, \omega)=\frac{1}{2}\left\langle A, \Delta_{a} A\right\rangle-\frac{1}{2} a\langle B, B\rangle-\left\langle\lambda, R \partial^{*} A\right\rangle-\langle\omega, Q A-B\rangle, \quad R \lambda=\lambda$,

where

$$
\Delta_{a}=\partial^{*} \partial+\partial R \partial^{*}+Q^{*} a Q=\Delta-\partial P \partial^{*}+Q^{*} a Q
$$

and the operator $Q$ is given by

$$
(Q A)(b)=\left(Q_{j} A\right)(b) \text { for } b \in \Lambda_{j}, \quad\left(Q_{0} A\right)(b)=A(b) .
$$

Of course the functional $h$ is equal to (2.5) for configurations $A$ satisfying (2.6), (2.12). The operator $Q^{*} a Q$ is defined by a formula similar to (2.14) with $a_{j}$ replaced simply by $a$, the other changes are obvious. To find the minimal configuration $A$ we have to solve the equations

$$
\begin{gathered}
\frac{\delta h}{\delta A}=\Delta_{a} A-\partial R \lambda-Q^{*} \omega=0, \\
\frac{\delta h}{\delta \lambda}=-R \partial^{*} A=0, \quad \frac{\delta h}{\delta \omega}=-(Q A-B)=0 .
\end{gathered}
$$

One of our main results will be that the operator $\Delta_{a}$ is bounded from below by a positive constant, hence the first equation implies

$$
A=G \partial R \lambda+G Q^{*} \omega, \quad G=\Delta_{a}^{-1} .
$$

The second and third equations can be written as

$$
R \partial^{*} G \partial R \lambda+R \partial^{*} G Q^{*} \omega=0, \quad Q G \partial R \lambda+Q G Q^{*} \omega=B .
$$

We investigate these two equations, and especially the operators $R \partial^{*} G \partial R$ and $R \partial^{*} G Q^{*}$.

At first we will write an integral representation of the operator $R$, analogous to the representation (1.27). By the formula (2.17) and properties of Gaussian integrals we have

$$
e^{-1 / 2\langle f, R f\rangle}=e^{-1 / 2\|f\|^{2}}\left(Z^{\prime-1} \int d \lambda \delta\left(Q^{\prime} \lambda\right) e^{-1 / 2\|f-\Delta \lambda\|^{2}}\right)^{-1}
$$

or

$$
e^{1 / 2\langle f, R f\rangle}=Z^{\prime-1} \int d \lambda \delta\left(Q^{\prime} \lambda\right) e^{-1 / 2\|\Delta \lambda\|^{2}+\langle\Delta f, \lambda\rangle} .
$$

Let us denote by $\mathscr{G}$ a covariance of the Gaussian integral on the right-hand side above. Thus we have

$$
R=\Delta \mathscr{G} \Delta
$$


It is easy to see that

$$
\mathscr{G}=G^{\prime 2}-G^{\prime 2} Q^{*}\left(Q^{\prime} G^{\prime 2} Q^{*}\right)^{-1} Q^{\prime} G^{\prime 2} .
$$

This formula, the equality (2.26) and the equalities $Q^{\prime} \mathscr{G}=\mathscr{G} Q^{*}=0$ imply the representation (2.17).

Let us consider the operator $R \partial^{*} G \partial R$. We have

$$
\begin{aligned}
e^{1 / 2\left\langle f, R \partial^{*} G \partial R f\right\rangle}= & Z^{-1} \int d A e^{-1 / 2\left\langle A, \Delta_{a} A\right\rangle+\left\langle f, R \hat{o}^{*} A\right\rangle} \\
= & Z^{-1} \int d A e^{-1 / 2\|\partial A\|^{2}-1 / 2 a\|Q A\|^{2}} e^{-1 / 2\left\|\partial^{*} A\right\|^{2}+\left\langle f, R \hat{o}^{*} A\right\rangle} \\
& \cdot Z^{\prime-1}\left(\int d \lambda \delta\left(Q^{\prime} \lambda\right) e^{-1 / 2\left\|\partial^{*} A-\Delta \lambda\right\|^{2}}\right)^{-1} .
\end{aligned}
$$

Applying the Faddeev-Popov procedure we change the Gaussian gauge fixing term in this integral into the $\delta$-function $\delta_{R}\left(R \partial^{*} A\right)$. Let us recall that $\delta_{R}$ is a $\delta$-function at the origin of the real, finite-dimensional Hilbert space $R$. We have

$$
\left|\operatorname{det}\left(\Delta \uparrow_{N\left(Q^{\prime}\right)}\right)\right| \int d \lambda^{\prime} \delta\left(Q^{\prime} \lambda\right) \delta_{R}\left(R \partial^{*} A+\Delta \lambda^{\prime}\right)=1
$$

(see the proof of the formula (1.46) in [4]) and we insert the expression above into the integral in (2.28). We change the order of integrations $\int d A \int d \lambda^{\prime} \ldots$ $=\int d \lambda^{\prime} \int d A \ldots$, next we make the gauge transformation $A \rightarrow A^{\lambda^{\prime}}=A-\partial \lambda^{\prime}$, and we again change the order of the integrations. Because $Q^{\prime} \lambda^{\prime}=0$, hence $Q A^{\lambda^{\prime}}=Q A$ $-\partial_{1} Q^{\prime} \lambda^{\prime}=Q A$, and we get

$$
\begin{aligned}
& e^{1 / 2\left\langle f, R \partial^{*} G \partial R f\right\rangle} \\
&=Z^{-1}\left|\operatorname{det}\left(\Delta \uparrow_{N\left(Q^{\prime}\right)}\right)\right| Z^{\prime} \int d A e^{-1 / 2\|\partial A\|^{2}-1 / 2 a\|Q A\|^{2}} \delta_{R}\left(R \partial^{*} A\right) \\
& \cdot \int d \lambda^{\prime} \delta\left(Q^{\prime} \lambda^{\prime}\right) e^{-1 / 2\left\|\partial^{*} A-\Delta \lambda^{\prime}\right\|^{2}+\left\langle f, R \partial^{*} A-\Delta \lambda^{\prime}\right\rangle}\left(\int d \lambda \delta\left(Q^{\prime} \lambda\right) e^{-1 / 2\left\|\partial^{*} A-\Delta \lambda^{\prime}-\Delta \lambda\right\|^{2}}\right)^{-1} \\
&= Z^{-1}\left|\operatorname{det}\left(\Delta \uparrow_{N\left(Q^{\prime}\right)}\right)\right| Z^{\prime} \int d A e^{-1 / 2\|\partial A\|^{2}-1 / 2 a\|Q A\|^{2}} \delta_{R}\left(R \partial^{*} A\right) \\
& \cdot \int d \lambda^{\prime} \delta\left(Q^{\prime} \lambda^{\prime}\right) e^{-1 / 2\left\|\Delta \lambda^{\prime}\right\|^{2}+\left\langle\Delta \partial^{*} A, \lambda^{\prime}\right\rangle-\left\langle\Delta f, \lambda^{\prime}\right\rangle}\left(\int d \lambda \delta\left(Q^{\prime} \lambda\right) e^{-1 / 2\|\Delta \lambda\|^{2}+\left\langle\Delta \partial^{*} A, \lambda\right\rangle}\right)^{-1} \\
&= Z^{-1}\left|\operatorname{det}\left(\Delta \uparrow_{N\left(Q^{\prime}\right)}\right)\right| Z^{\prime} \int d A e^{-1 / 2\|\partial A\|^{2}-1 / 2 a\|Q A\|^{2}} \delta_{R}\left(R \partial^{*} A\right) \\
& \cdot e^{-\left\langle\Delta \partial^{*} A, \mathscr{G} \Delta f\right\rangle+1 / 2\langle\Delta f, \mathscr{G} \Delta\rangle}=e^{1 / 2\langle f, R f\rangle} .
\end{aligned}
$$

The last equality follows from the identity (2.26) and from the presence of the $\delta$-function $\delta_{R}\left(R \partial^{*} A\right)$. Thus

$$
R \partial^{*} G \partial R=R .
$$

Now we consider the operator $R \partial^{*} G Q^{*}$. We have

$$
R \partial^{*} G Q^{*}=Z^{-1} \int d A e^{-1 / 2\left\langle A, \Delta_{a} A\right\rangle} R \partial^{*} A Q A .
$$

Applying the same operations as in (2.28)-(2.30) we get,

$$
\begin{aligned}
R \partial^{*} G Q^{*}= & Z^{-1}\left|\operatorname{det}\left(\Delta \uparrow_{N\left(Q^{\prime}\right)}\right)\right| Z^{\prime} \int d A e^{-1 / 2\|\partial A\|^{2}-1 / 2 a\|Q A\|^{2}} \\
& \cdot \delta_{R}\left(R \partial^{*} A\right) \int d \lambda^{\prime} \delta\left(Q^{\prime} \lambda\right) e^{-1 / 2\left\|\partial^{*} A-\Delta \lambda^{\prime}\right\|^{2}}\left(R \partial^{*} A-\Delta \lambda^{\prime}\right) Q A \\
& \cdot\left(\int d \lambda \delta\left(Q^{\prime} \lambda\right) e^{-1 / 2\left\|\partial^{*} A-\Delta \lambda\right\|^{2}}\right)^{-1} \\
= & Z^{-1}\left|\operatorname{det}\left(\Delta \uparrow_{N\left(Q^{\prime}\right)}\right)\right| Z^{\prime} \int d A e^{-1 / 2\|\partial A\|^{2}-1 / 2 a\|Q A\|^{2}} \delta_{R}\left(R \partial^{*} A\right) \\
& \cdot \int d \lambda^{\prime} \delta\left(Q^{\prime} \lambda\right) e^{-1 / 2\left\|\Delta \lambda^{\prime}\right\|^{2}}\left(-\Delta \lambda^{\prime}-\Delta \mathscr{G} \Delta \partial^{*} A\right) Q A \\
& \cdot\left(\int d \lambda \delta\left(Q^{\prime} \lambda\right) e^{-1 / 2\|\Delta \lambda\|^{2}}\right)^{-1}=0 .
\end{aligned}
$$


We have applied the translation $\lambda^{\prime} \rightarrow \lambda^{\prime}+\mathscr{G} \Delta \partial^{*} A$, and the same for $\lambda$, to get the second equality. The last follows again from (2.26). Hence we have

$$
R \partial^{*} G Q^{*}=0, \quad Q G \partial R=0 .
$$

The equalities (2.31), (2.34) are generalizations of the equalities (1.97), (1.95) in [4] and can be applied now to Eqs. (2.23). The first equation is simply $R \lambda=\lambda=0$, and the second is $Q G Q^{*} \omega=B$. The operator $G$ is positive, hence $Q G Q^{*}$ is positive also and an inverse is a well-defined and positive operator. We get $\omega=\left(Q G Q^{*}\right)^{-1} B$, and from (2.22) we obtain finally

$$
A=H B=G Q^{*}\left(Q G Q^{*}\right)^{-1} B .
$$

Thus we have proved that there exists exactly one solution of the equations (2.21), hence exactly one critical configuration of (2.5) satisfying (2.6), (2.12), and given by (2.35). The only assumption we have used was the positivity of the operator $\Delta_{a}$, $a>0$, or $G$. This will be proven later. We have obtained the same representation for the operator $H$ as in (1.103), hence properties of this operator are related to corresponding properties of $G$. We have also the representation (1.107) for the projection operator $P$.

The variational problem $(2.5),(2.6),(2.12)$ can be solved also in a different way. We use the fact that the configuration $A$ is fixed outside $\Omega_{1}$ by the conditions (2.6), thus we should obtain a solution of the problem using operators chosen arbitrarily there, especially operators with arbitrary boundary conditions outside $\Omega_{1}$. Let us take a neighbourhood $\Omega$ of the domain $\Omega_{1}$. We assume that it is a sum of big blocks of the lattice $T_{1}$. For example we may take $\Omega=$ a sum of big blocks of the lattice $T_{1}$ with distances to $\left.\Omega_{1} \leqq R M\right\}$. We will consider operators with Dirichlet boundary conditions on $\Omega^{c}$. For the quadratic form (2.5) we have by (2.6),

$$
\frac{1}{2}\left\langle A, \partial^{*} \partial A\right\rangle=\frac{1}{2}\left\langle A, \Omega \partial^{*} \partial \Omega A\right\rangle+\left\langle A, \Omega \partial^{*} \partial \Omega^{c} B\right\rangle+\frac{1}{2}\left\langle B, \Omega^{c} \partial^{*} \partial \Omega^{c} B\right\rangle,
$$

hence the Lagrange function can be taken as equal to

$$
\begin{aligned}
h(A, \lambda, \omega)= & \frac{1}{2}\left\langle A, \Omega \Delta_{a} \Omega A\right\rangle+\left\langle A, \Omega \partial^{*} \partial \Omega^{c} B\right\rangle+\frac{1}{2}\left\langle B, \Omega^{c} \partial^{*} \partial \Omega^{c} B\right\rangle \\
& -\frac{1}{2}\langle\Omega B, a \Omega B\rangle-\left\langle\lambda, R \partial^{*} A\right\rangle-\langle\omega, Q A-B\rangle,
\end{aligned}
$$

$R \lambda=\lambda, \omega=0$ on $\Omega^{c}$. We may take also $R$ defined by operators with Dirichlet boundary conditions on $\Omega^{c}$. Solving critical point equations for $h(A, \lambda, \omega)$ and denoting $G(\Omega)=\left(\Delta_{a} \uparrow_{\Omega}\right)^{-1}=\left(\Omega \Delta_{a} \Omega\right)^{-1}$, we get

$$
\begin{aligned}
A= & G(\Omega) Q^{*}\left(Q G(\Omega) Q^{*}\right)^{-1} B+G(\Omega) Q^{*}\left(Q G(\Omega) Q^{*}\right)^{-1} Q G(\Omega) \partial^{*} \partial \Omega^{c} B \\
& -G(\Omega) \partial^{*} \partial \Omega^{c} B .
\end{aligned}
$$

We have to notice only that the equalities (2.31), (2.34) hold for the operator $G(\Omega)$ also. If $B=0$ outside $\Omega_{1}$, then the above representation simplifies and we get (2.35) with $G(\Omega)$ instead of $G$.

All the reasonings and the results of this paper hold, with minor and obvious changes, for the operators $G(\Omega)$. We choose $\Omega=T_{\eta}$ for simplicity of notation.

Let us make a remark about the formulation of the problem (2.5), (2.6), (2.12) and the geometric setting (2.1), (2.2). Such problems arise in a natural way when the 
so-called "large-fields regions" are considered, see the paper [2]. We will use them also systematically as a convenient way of providing boundary conditions for operators such as $\Delta_{a}$. If we are interested in a Green's function for this operator considered on a domain $\Omega \subset T_{\eta}$, we have to introduce some boundary conditions. We can do it taking a sequence (2.1) with $\Omega=\Omega_{k}$ and smallest possible domains $B^{j}\left(\Lambda_{j}\right)$, and considering the operator $\Delta_{a}$ defined by (2.19), (2.20) for this sequence. Boundary conditions of this type can be interpreted as obtained by building an effective mass on the domain $\Omega_{1} \backslash \Omega_{k}$, starting from $O(1)$ on $\Omega_{k}$ up to $+\infty$ outside $\Omega_{1}$. They are technically much more convenient than the usual local boundary conditions, like Dirichlet or Neumann boundary conditions, which give unpleasant singularities connected with sharp edges of the domain $\Omega$.

The main goal of this paper is to investigate the properties of the operators $G^{\prime}$, $G, Q^{\prime} G^{\prime 2} Q^{*}, Q G Q^{*}$. We will apply the methods and the results of the previous paper. The basic tools are the generalized random walk expansions. Each set $\Lambda_{j}$ is a sum of big blocks of the size $M L^{j} \eta\left(\Lambda_{j} \subset T_{L^{j} \eta}^{(j)}\right)$, or of the size $M$ if $\Lambda_{j}$ is scaled to unit lattice. We cover $B^{j}\left(\Lambda_{j}\right)$ by a sum of cubes $\square$ of the size $2 M L^{j} \eta$, each cube being a sum of $2^{d}$ big blocks with a center $y \in \Lambda_{j}$ (more exactly it belongs to the boundary of this set also). Taking these covers for all $j$ from 0 to $k$ we get a family $\mathscr{D}$ of cubes $\square$

of different sizes and such that $T_{\eta}=\bigcup_{\square \in \mathscr{D}} \square$. We will identify this family of cubes with the set of centers of these cubes. We construct also the corresponding family of functions $h$ described in (1.118), and rescale them to proper scales. They satisfy

$$
\sum_{\square \in \mathscr{D}} h_{\square}^{2}=1
$$

\section{B. Operators Defining Gauge Conditions}

Let us consider at first the operator $G^{\prime}$. We construct a random walk expansion of it in a similar way as in [3]. We define

$$
G_{0}^{\prime}=\sum_{\square} h_{\square} G^{\prime}(\square) h_{\square},
$$

where $G^{\prime}(\square)$ is an inverse of $\Delta_{a}^{\prime}$ with some boundary conditions on the boundary of $\square$, e.g. with Neumann boundary conditions as in [3]. Repeating the calculations in the paper we get

$$
\Delta_{a}^{\prime} G_{0}^{\prime}=I-\sum_{\square} K\left(h_{\square}\right) G^{\prime}(\square) h_{\square}=I-R,
$$

where

$$
\begin{aligned}
(K(h) \lambda)(x)= & \sum_{b \in s t(x)}(\partial h)(b)(\partial \lambda)(b)-(\Delta h)(x) \lambda(x) \\
& -a_{j}\left(L^{j} \eta\right)^{-2} \sum_{x^{\prime} \in B^{j}\left(y^{j}(x)\right)} L^{-j d}(\partial h)\left(\Gamma_{x, y^{j}(x), x^{\prime}}^{(j)}\right) \lambda\left(x^{\prime}\right) \\
& \text { if } \quad x \in B^{j}\left(\Lambda_{j}\right) .
\end{aligned}
$$

The derivatives above are on $\eta$-scale. Let us estimate one term in the sum over $\square$ in (2.38). Let us assume that $\square$ is a cube connected with a $L^{j} \eta$-scale (i.e. a cube of the 
size $\left.2 M L^{j} \eta\right)$ and intersecting maybe the domain $B^{j+1}\left(\Lambda_{j+1}\right)$. Rescaling all the expressions in $K\left(h_{\square}\right) G^{\prime}(\square) h_{\square} \lambda$ to $L^{-j}$-scale, we get

$$
\begin{aligned}
& \left(K\left(h_{\square}\right) G^{\prime}(\square) h_{\square} \lambda\right)\left(L^{j} \eta x\right) \\
& =\sum_{b \in s t(x)}\left(\partial^{L^{-j}} h_{\square}\right)(b)\left(\partial^{L^{-j}} G^{\prime}(\square) h_{\square} \lambda\right)(b)-\left(\Delta^{L^{-j}} h_{\square}\right)(x)\left(G^{\prime}(\square) h_{\square} \lambda\right)(x) \\
& \quad-a_{j} \sum_{x^{\prime} \in B^{j}\left(y^{j}(x)\right)} L^{-j d}\left(\partial^{L^{-j}} h_{\square}\right)\left(\Gamma_{x, y^{j}(x), x^{\prime}}^{(j)}\right)\left(G^{\prime}(\square) h_{\square} \lambda\right)\left(x^{\prime}\right) \\
& \quad \text { if } \quad x \in B^{j}\left(\Lambda_{j}\right) \subset T_{L^{-j}},
\end{aligned}
$$

or the last term above is replaced by a term defined in the same way but with $j+1$ instead of $j$ and with the additional factor $L^{-2}$ if $x \in B^{j+1}\left(\Lambda_{j+1}\right) \subset T_{L^{-j}}$. Of course the operator $G^{\prime}(\square)$ is defined on $L^{-j}$-scale, hence

$$
\left(G^{\prime}(\square) h_{\square} \lambda\right)(x)=\sum_{x^{\prime}} L^{-j d} G^{\prime}\left(\square ; x, x^{\prime}\right) h_{\square}\left(x^{\prime}\right) \lambda\left(x^{\prime}\right) .
$$

If $\square \subset B^{j}\left(\Lambda_{j}\right)$, then the necessary properties of $G^{\prime}(\square)$ are described in Lemma 2.2 [3]. If $\square$ intersects both domains $B^{j}\left(\Lambda_{j}\right)$ and $B^{j+1}\left(\Lambda_{j+1}\right)$, then we express $G^{\prime}(\square)$ in terms of operators introduced in the paper. On the basis of the formulas (2.12), (2.13) [1] we have

$$
\begin{aligned}
e^{1 / 2\left\langle f, G^{\prime}(\square) f\right\rangle}= & Z^{-1} \int d \lambda \exp \left[-1 / 2\left\langle\lambda,\left(\Delta_{\square}^{L^{-j}, N}+Q^{*} a Q^{\prime} \uparrow_{\square}\right) \lambda\right\rangle+\langle\lambda, f\rangle\right] \\
= & Z^{\prime-1} \int d \omega \uparrow_{\Lambda} \exp \left[-1 / 2 a L^{d-2} \sum_{y \in \Lambda^{\prime}}\left|\left(Q^{\prime} \omega\right)(y)\right|^{2}\right] \\
& \cdot \int d \lambda \exp \left[-1 / 2 a_{j}\left\|\omega-Q_{j}^{\prime} \lambda\right\|^{2}-1 / 2\left\langle\lambda, \Delta_{\square}^{L-j, N} \lambda\right\rangle+\langle\lambda, f\rangle\right],
\end{aligned}
$$

where $\Lambda=\square^{(j)} \cap B\left(\Lambda_{j+1}\right)$ and $\omega$ is equal to 0 beyond $\Lambda$. Further, we have after the translation $\lambda \rightarrow \lambda+\mathrm{G}_{j}^{\prime}(\square) Q_{j}^{*} \omega$

$$
\begin{aligned}
e^{1 / 2\left\langle f, G^{\prime}(\square) f\right\rangle} & \\
= & Z^{\prime-1} \int d \omega \uparrow_{\Lambda} \exp \left[-1 / 2 a L^{-2}\left\langle\omega, Q^{*} Q^{\prime} \omega\right\rangle-1 / 2 a_{j}\|\omega\|^{2}\right. \\
& \left.+1 / 2 a_{j}^{2}\left\langle\omega, Q_{j}^{\prime} G_{j}^{\prime}(\square) Q_{j}^{\prime *} \omega\right\rangle+\left\langle\omega, Q_{j}^{\prime} G_{j}^{\prime}(\square) f\right\rangle\right] \\
& \cdot \int d \lambda \exp \left[-1 / 2\left\langle\lambda,\left(G_{j}^{\prime}(\square)\right)^{-1} \lambda\right\rangle+\langle\lambda, f\rangle\right] \\
= & e^{1 / 2\left\langle f, G_{j}^{\prime}(\square) f\right\rangle} Z^{\prime-1} \int d \omega \uparrow_{\Lambda} \exp \left[-1 / 2\left\langle\omega,\left(C_{\Lambda}^{(j)}(\square)\right)^{-1} \omega\right\rangle+\left\langle\omega, Q_{j}^{\prime} G_{j}^{\prime}(\square) f\right\rangle\right] \\
= & \exp \left[1 / 2\left\langle f, G_{j}^{\prime}(\square) f\right\rangle+\left\langle f, G_{j}^{\prime}(\square) Q_{j}^{*} C_{\Lambda}^{(j)}(\square) Q_{j}^{\prime} G_{j}^{\prime}(\square) f\right\rangle\right] .
\end{aligned}
$$

Properties of the operators $G_{j}^{\prime}(\square), G_{j}^{\prime}(\square) Q_{j}^{* *}$ and $C_{A}^{(j)}(\square)$ are described in Lemmas 2.2, 2.4, Proposition 2.3 [3]. From these and (2.42) we get

$$
\left|\left(G^{\prime}(\square) \lambda\right)(x)\right|, \quad\left|\left(\partial_{\mu}^{L^{-j}} G^{\prime}(\square) \lambda\right)(x)\right| \leqq O(1) e^{-\delta_{0} \operatorname{dist}(x, \operatorname{supp} \lambda)}|\lambda| .
$$

This inequality and (2.40) imply

$$
\left|\left(K\left(h_{\square}\right) G^{\prime}(\square) h_{\square} \lambda\right)(x)\right| \leqq O\left(M^{-1}\right) e^{-\delta_{0}|x-y|}|\lambda|
$$

if either $\operatorname{supp} \lambda \subset B^{j}(y)$ for $y \in \Lambda_{j}$, or $\operatorname{supp} \lambda \subset B^{j+1}(y)$ for $y \in \Lambda_{j+1}$. The distance in the above inequality is measured on $L^{-j}$-scale.

The inequality (2.44) implies an inequality for the operator $R$. To write it we will introduce a new definition of distance, taking into account that natural scales 
are different on different subdomains $B^{j}\left(\Lambda_{j}\right)$ of $T_{\eta}$. Let us denote

$$
\mathfrak{B}=\bigcup_{j=0}^{k} \Lambda_{j}
$$

We will identify this set with the set of corresponding blocks. For an arbitrary contour $\Gamma$ on the lattice $T_{\eta}$ we put $|\Gamma|=n \eta$, where $n$ is a number of bonds the contour $\Gamma$ consists of. We will define a new distance between two points of $\mathfrak{B}$. We consider a special class of contours $\Gamma$. They have the property that a part of $\Gamma$ contained in $B^{j}\left(\Lambda_{j}\right)$ consists of bonds of the lattice $\Lambda_{j}$. Now we define

$$
d\left(y, y^{\prime}\right)=\inf _{\Gamma_{y, y^{\prime}}} \sum_{j=0}^{k}\left(L^{j} \eta\right)^{-1}\left|\Gamma_{y, y^{\prime}} \cap B^{j}\left(\Lambda_{j}\right)\right|, \quad y, y^{\prime} \in \mathfrak{B},
$$

where the infimum is taken over all admissible contours described above, with endpoints, $y, y^{\prime}$. We may extend this definition and define the distance for a pair of arbitrary points $x, \quad x^{\prime} \in T_{\eta}$ putting $d\left(x, x^{\prime}\right)=d\left(y^{j}(x), y^{j^{\prime}}\left(x^{\prime}\right)\right)$ if $x \in B^{j}\left(\Lambda_{j}\right)$, $x^{\prime} \in B^{j^{\prime}}\left(\Lambda_{j^{\prime}}\right)$. We need some bounds for the distance $d\left(y, y^{\prime}\right)$. It is convenient to introduce new notations. The domain $\Omega_{j}$ is a sum of cubes of the size $M L^{j} \eta$. A sum of faces of these cubes which are not contained in the interior of $\Omega_{j}$ forms a surface. We denote this surface by $\Sigma_{j}$ and we use the same notation for the set $T^{(j)} \cap \Sigma_{j}=\Lambda_{j}$ $\cap \Sigma_{j}$. The surface $\Sigma_{j}$ separates the sets $B^{j}\left(\Lambda_{j}\right)$ and $B^{j-1}\left(\Lambda_{j-1}\right)$. Now let us consider the definition (2.46). Of course the infimum is attained at some contour $\Gamma_{y, y^{\prime}}$. Let us assume that $y \in \Lambda_{j}, y^{\prime} \in \Lambda_{j^{\prime}}$. We will analyze a structure of the contour $\Gamma_{y, y^{\prime}}$ and we will obtain a bound for the distance $d\left(y, y^{\prime}\right)$. The contour $\Gamma_{y, y^{\prime}}$ starts at $y$ and intersects either the surface $\Sigma_{j}$, or the surface $\Sigma_{j+1}$, the first time at a point $y_{1}$. Let us denote the index of the surface by $j_{1}$, so $y_{1} \in \Sigma_{j_{1}}$. A next portion of the contour $\Gamma_{y_{1}, y^{\prime}}$ starting at $y_{1}$ is contained in one of the domains $B^{j_{1}}\left(\Lambda_{j_{1}}\right), B^{j_{1}-1}\left(\Lambda_{j_{1}-1}\right)$, and intersects one of the surfaces $\Sigma_{j_{1}-1}, \Sigma_{j_{1}}, \Sigma_{j_{1}+1}$ the first time at a point $y_{1}^{\prime}$. If this point belongs to $\Sigma_{j_{1}}$, then we consider a next portion of the contour in the same way and we denote by $y_{1}^{\prime}$ the last intersection point of the contour $\Gamma_{y_{1}, y^{\prime}}$ with the surface $\Sigma_{j_{1}}$. More exactly it is such a point of the contour $\Gamma_{y_{1}, y^{\prime}}$ that $y_{1}^{\prime} \in \Sigma_{j_{1}}$, the portion $\Gamma_{y_{1}, y_{1}^{\prime}}$ of the contour does not intersect any surface $\Sigma_{j}$ with $j \neq j_{1}$, and it is the last point of the contour with this property. If the point belongs to one of the surfaces $\Sigma_{j_{1}-1}, \Sigma_{j_{1}+1}$, then we denote it by $y_{2}$ and we denote the index of the surface by $j_{2}$. For uniformity we take $y_{1}^{\prime}=y_{1}$ in this case and we define $\Gamma_{y_{1}, y_{1}^{\prime}}$ as consisting of one point. Thus the portion $\Gamma_{y_{1}^{\prime}, y_{2}}$ of the contour $\Gamma_{y_{1}, y^{\prime}}$ connects the surface $\Sigma_{j_{1}}$ with the surface $\Sigma_{j_{2}}$ and is contained in $B^{j_{12}}\left(\Lambda_{j_{12}}\right), j_{12}=\min \left\{j_{1}, j_{2}\right\}$, moreover $\left|j_{1}-j_{2}\right|$ $=1$. Now we consider the contour $\Gamma_{y_{2}, y^{\prime}}$ and we apply the same analysis. Continuing this way we obtain a sequence of points $y, y_{1}, y_{1}^{\prime}, y_{2}, y_{2}^{\prime}, \ldots, y_{m}, y_{m}^{\prime}, y^{\prime}$ on $\Gamma_{y, y^{\prime}}$, and a sequence of indices $j, j_{1}, j_{2}, \ldots, j_{m}, j^{\prime}$ with the following properties:

$$
\Gamma_{y, y^{\prime}}=\Gamma_{y, y_{1}} \cup \bigcup_{l=1}^{m}\left(\Gamma_{y_{l}, y_{i}^{\prime}} \cup \Gamma_{y_{i}^{\prime}, y_{l+1}}\right), \quad y_{m+1}=y^{\prime}
$$

$y_{l}, y_{l}^{\prime} \in \Sigma_{j_{l}}, \Gamma_{y_{l}, y_{l}^{\prime}}$ does not intersect any other surface $\Sigma_{j}, j \neq j_{l}$, $\Gamma_{y^{\prime}, y_{l+1}}$ connects the surface $\Sigma_{j_{l}}$ with the surface $\Sigma_{j_{l+1}}$ and is contained in $B^{j_{l, l+1}}\left(\Lambda_{j_{l, l+1}}\right)$, where $j_{l, l+1}=\min \left\{j_{l}, j_{l+1}\right\}$, $\left|j_{l}-j_{l+1}\right|=1$. 
From this decomposition and the definition (2.46) we get

$$
\begin{aligned}
d\left(y, y^{\prime}\right) \geqq & \left(L^{j} \eta\right)^{-1}\left|\Gamma_{y, y_{1}}\right|+\sum_{l=1}^{m}\left(L^{j_{l}} \eta\right)^{-1}\left|\Gamma_{y_{l}, y_{l}^{\prime}}\right| \\
& +\sum_{l=1}^{m}\left(L^{j_{l, l+1}} \eta\right)^{-1}\left|\Gamma_{y^{\prime}, y_{l+1}}\right| \\
\geqq & \left(L^{j} \eta\right)^{-1}\left|y-y_{1}\right|+\sum_{l=1}^{m}\left(L^{j_{l}} \eta\right)^{-1}\left|y_{l}-y_{l}^{\prime}\right| \\
& +\sum_{l=1}^{m}\left(L^{j_{l, l+1}} \eta\right)^{-1}\left|y_{l}^{\prime}-y_{l+1}\right| .
\end{aligned}
$$

Now let us come back to the inequality (2.44) and its consequences. One of them follows from the equality (2.38) where the operator $R$ was defined. We get

$$
|R \lambda| \leqq O\left(M^{-1}\right)|\lambda|,
$$

thus the operator $R$ has a small norm in the space $L^{\infty}$ for $M$ sufficiently large, and we get

$$
\begin{aligned}
G^{\prime} & =G_{0}^{\prime}(I-R)^{-1}=G_{0}^{\prime} \sum_{n=0}^{\infty} R^{n} \\
& =\sum_{\omega} h_{\square_{0}} G^{\prime}\left(\square_{0}\right) h_{\square_{0}} K\left(h_{\square_{1}}\right) G^{\prime}\left(\square_{1}\right) h_{\square_{1}} \cdot \ldots \cdot K\left(h_{\square_{n}}\right) G^{\prime}\left(\square_{n}\right) h_{\square_{n}},
\end{aligned}
$$

where

$$
\omega=\left(\square_{0}, \square_{1}, \ldots, \square_{n}\right), \quad \square_{i} \in \mathscr{D}, \quad \square_{i} \cap \square_{i+1} \neq \emptyset .
$$

Both series above are convergent in the space $L^{\infty}$, and in the Hölder norm $\|\cdot\|_{1, \alpha}$ also. To investigate properties of $G^{\prime}$, and especially an exponential decay, we will use another bound for the operator $R$. From (2.44) and (2.38) we have

$$
|(R \lambda)(x)| \leqq O\left(M^{-1}\right) e^{-\delta_{0} d(x, y)}|\lambda| \quad \text { if } \quad \operatorname{supp} \lambda \subset B^{j}(y), \quad y \in \Lambda_{j} .
$$

Of course in the considered case the operator $R$ is short-ranged and for many points $x$ and $y$ the property (2.51) holds in void, but the point is that this property is preserved under the composition of operators possessing it. A summation preserves it also, so we will have it for the operator $G^{\prime}$. Let us consider $n$ operators $R_{1}, R_{2}, \ldots, R_{n}$ satisfying (2.51) with a constant $O(1)$ instead of $O\left(M^{-1}\right)$, and let us consider the composition $R=R_{1} R_{2} \cdot \ldots \cdot R_{n}$. We consider $(R \lambda)(x)$ for $x \in B^{j}(y)$, $y \in \Lambda_{j}$ and $\operatorname{supp} \lambda \subset B^{j^{\prime}}\left(y^{\prime}\right), y^{\prime} \in \Lambda_{j^{\prime}}$. We have

$$
(R \lambda)(x)=\sum_{y_{1}, y_{2}, \ldots, y_{n-1} \in \mathfrak{B}}\left(R_{1} \Delta\left(y_{1}\right) R_{2} \Delta\left(y_{2}\right) \cdot \ldots \cdot R_{n-1} \Delta\left(y_{n-1}\right) R_{n} \lambda\right)(x),
$$

where $\Delta(y)=B^{j}(y)$ if $y \in \Lambda_{j}$, hence

$$
|(R \lambda)(x)| \leqq O(1)^{n} \sum_{y_{1}, y_{2}, \ldots, y_{n-1} \in \mathfrak{B}} e^{-\delta_{0} d\left(y, y_{1}\right)} e^{-\delta_{0} d\left(y_{1}, y_{2}\right)} \cdot \ldots \cdot e^{-\delta_{0} d\left(y_{n-1}, y^{\prime}\right)}|\lambda| .
$$

The distances $d\left(y_{i}, y_{i+1}\right)$ are determined by some admissible contours $\Gamma_{y_{i}, y_{i+1}}$. Taking the contour $\Gamma_{y, y^{\prime}}=\bigcup_{i=0}^{n-1} \Gamma_{y_{i}, y_{i+1}}$, where $y_{0}=y, y_{n}=y^{\prime}$, we obtain an 
admissible contour with end-points $y, y^{\prime}$, hence by definition (2.46) we have

$$
d\left(y, y_{1}\right)+d\left(y_{1}, y_{2}\right)+\ldots+d\left(y_{n-1}, y^{\prime}\right) \geqq d\left(y, y^{\prime}\right) .
$$

This is of course the triangle inequality for our distance. It implies

$$
|(R \lambda)(x)| \leqq O(1)^{n} e^{-(1-\alpha) \delta_{0} d\left(y, y^{\prime}\right)} \sum_{y_{1}, \ldots, y_{n-1} \in \mathfrak{B}} e^{-\alpha \delta_{0} d\left(y, y_{1}\right)} \cdot \ldots \cdot e^{-\alpha \delta_{0} d\left(y_{n-1}, y^{\prime}\right)}|\lambda|
$$

with arbitrary $0<\alpha<1$. To estimate the sum above we add a summation over $y^{\prime} \in \mathfrak{B}$ and we get

$$
\sum_{y_{1}, \ldots, y_{n-1} \in \mathfrak{B}} e^{-\alpha \delta_{0} d\left(y, y_{1}\right)} \cdot \ldots \cdot e^{-\alpha \delta_{0} d\left(y_{n-1}, y^{\prime}\right)} \leqq\left(\sup _{y \in \mathfrak{B}} \sum_{y^{\prime} \in \mathfrak{B}} e^{-\alpha \delta_{0} d\left(y, y^{\prime}\right)}\right)^{n} .
$$

Thus we have to estimate the sum on the right-hand side above for one exponential factor. We use the inequality (2.48). From the condition (2.2) and from the definition of the points $y_{l}^{\prime}, y_{l+1}$, more exactly from the fact that they belong to different surfaces $\Sigma_{j}$, we have

$$
\left(L^{j_{l, l+1}} \eta\right)^{-1}\left|y_{l}^{\prime}-y_{l+1}\right|>R M .
$$

The distances on the right-hand side of (2.48) are scaled to unit lattice and we estimate $e^{-\alpha \delta_{0} d\left(y, y^{\prime}\right)}$ using (2.48) and adding sums over all intermediate points $y_{l}, y_{l}^{\prime}$, $l=1, \ldots, m$, and over all possible numbers $m$. We get

$$
\begin{aligned}
\sum_{y^{\prime} \in \mathfrak{B}} e^{-\alpha \delta_{0} d\left(y, y^{\prime}\right)=} & \sum_{j^{\prime}=0}^{k} \sum_{y^{\prime} \in \Lambda_{j^{\prime}}} e^{-\alpha \delta_{0} d\left(y, y^{\prime}\right)} \\
\leqq & \sum_{j^{\prime}=0}^{k} \sum_{m=\max \left\{\left|j-j^{\prime}\right|-1,0\right\}}^{\infty} e^{-\frac{1}{2} \alpha \delta_{0} m R M} \sum_{z_{1}, z_{i}^{\prime}, l=1, \ldots, m, z_{m+1} \in Z^{d}} \\
& \cdot e^{-\frac{1}{2} \alpha \delta_{0}\left|s(y)-z_{1}\right|} e^{-\frac{1}{2} \alpha \delta_{0}\left|z_{1}-z_{1}^{\prime}\right|} \ldots \cdot e^{-\frac{1}{2} \alpha \delta_{0}\left|z_{m}^{\prime}-z_{m+1}\right|} \\
= & \sum_{j^{\prime}=0}^{k} \sum_{m=\max \left\{\left|j-j^{\prime}\right|-1,0\right\}}^{\infty} e^{-\frac{1}{2} \alpha \delta_{0} m R M}\left(c_{0}(1 / 2 \alpha)\right)^{d(2 m+1)}
\end{aligned}
$$

where $s(y)$ denotes a scaled image of $y$ on unit lattice, and

$$
c_{0}(\alpha)=\sum_{z \in Z} e^{-\alpha \delta_{0}|z|}<2\left(1-e^{-\alpha \delta_{0}}\right)^{-1}<\frac{4}{\alpha \delta_{0}} .
$$

Now we require that $R M$ is sufficiently large, i.e. we assume

$$
\frac{1}{4} \alpha \delta_{0} R M>2 d \log c_{0}\left(\frac{1}{2} \alpha\right)+1 .
$$

Then the sum over $m$ can be estimated by

$$
c_{0}^{d}\left(\frac{1}{2} \alpha\right) e^{-\frac{1}{4} \alpha \delta_{0} R M \max \left\{\left|j-j^{\prime}\right|-1,0\right\}} \sum_{m=0}^{\infty} e^{-m} \leqq 6 c_{0}^{d}\left(\frac{1}{2} \alpha\right) e^{-\left|j-j^{\prime}\right|} .
$$

The summation over $j^{\prime}$ gives finally the constant $12 c_{0}^{d}\left(\frac{1}{2} \alpha\right)=c_{1}(\alpha)$. For easy reference let us formulate these results in 
Lemma 2.1. For the numbers $\alpha, 0<\alpha<1, c_{1}(\alpha)=12 c_{0}^{d}\left(\frac{1}{2} \alpha\right)$, and $R M$ satisfying (2.59) we have

$$
\begin{gathered}
e^{-\alpha \delta_{0} d\left(y, y^{\prime}\right)} \leqq e^{-\alpha \delta_{0} R M \max \left\{\left|j-j^{\prime}\right|-1,0\right\}}, \quad y \in \Lambda_{j}, \quad y^{\prime} \in \Lambda_{j^{\prime}}, \\
\sup _{y \in \mathfrak{B}} \sum_{y^{\prime} \in \mathfrak{B}} e^{-\alpha \delta_{0} d\left(y, y^{\prime}\right)} \leqq c_{1}(\alpha),
\end{gathered}
$$

hence

$$
\sum_{y_{1}, \ldots, y_{n} \in \mathfrak{B}} e^{-\alpha \delta_{0} d\left(y, y_{1}\right)} \cdot \ldots \cdot e^{-\alpha \delta_{0} d\left(y_{n-1}, y_{n}\right)} \leqq c_{1}(\alpha)^{n}
$$

and

$$
\sum_{y_{1}, \ldots, y_{n-1} \in \mathfrak{B}} e^{-\delta_{0} d\left(y, y_{1}\right)} \cdot \ldots \cdot e^{-\delta_{0} d\left(y_{n-1}, y_{1}\right)} \leqq c_{1}(\alpha)^{n} e^{-(1-\alpha) \delta_{0} d\left(y, y^{\prime}\right)} .
$$

We apply these results e.g. for $\alpha=1 / 2$, then $c_{0}$ is an absolute constant and the condition (2.59) on RM can be written as $\delta_{0} R M>16 d \log c_{0}+1$, so it is a condition written in terms of absolute constants $\delta_{0}, c_{0}$. From the lemma and (2.55) we get

$$
|(R \lambda)(x)| \leqq O(1)^{n} c_{1}^{n} e^{-\frac{1}{2} \delta_{0} d\left(y, y^{\prime}\right)}|\lambda|, \quad x \in B^{j}(y), \quad \operatorname{supp} \lambda \subset B^{j^{\prime}}\left(y^{\prime}\right) .
$$

Applying this inequality to the $n^{\text {th }}$ power of the operator $R$ in (2.38) we have

$$
\left|\left(R^{n} \lambda\right)(x)\right| \leqq\left(O\left(M^{-1}\right) c_{1}\right)^{n} e^{-\frac{1}{2} \delta_{0} d\left(y, y^{\prime}\right)}|\lambda|,
$$

and this implies finally for $x \in B^{j}(y), \operatorname{supp} \lambda \subset B^{j^{\prime}}\left(y^{\prime}\right)$,

$$
\begin{aligned}
\left|\left(G^{\prime} \lambda\right)(x)\right| \leqq & \sum_{n=0}^{\infty}\left|\left(G_{0}^{\prime} R^{n} \lambda\right)(x)\right| \leqq O(1)\left(L^{j} \eta\right)^{2} \\
& \cdot \sum_{y^{\prime \prime}:\left(L^{j} \eta\right)^{-1}\left|y-y^{\prime \prime}\right| \leqq 2 d M} e^{-\delta_{0}\left(L^{j} \eta\right)^{-1}\left|y-y^{\prime \prime}\right|} \\
& \cdot \sum_{n=0}^{\infty}\left(O\left(M^{-1}\right) c_{1}\right)^{n} e^{-\frac{1}{2} \delta_{0} d\left(y^{\prime \prime}, y^{\prime}\right)}|\lambda| \\
\leqq & O(1)\left(L^{j} \eta\right)^{2} e^{-\frac{1}{2} \delta_{0} d\left(y, y^{\prime}\right)}|\lambda| .
\end{aligned}
$$

The similar inequalities hold for a derivative of $G^{\prime} \lambda$ and for a Hölder norm of a derivative, but with $\left(L^{j} \eta\right)^{2}$ replaced by $L^{j} \eta$ and $\left(L^{j} \eta\right)^{1-\alpha}$ correspondingly. Let us formulate these results in

Proposition 2.2. If we have (2.1), (2.2) and $M$ is sufficiently large, then the operator $G^{\prime}=\Delta_{a}^{\prime-1}(a=1)$ satisfies the inequalities

$$
\begin{aligned}
& \left|\left(G^{\prime} \lambda\right)(x)\right|, \quad\left|\left(\nabla G^{\prime} \lambda\right)(x)\right|, \quad\left|\left(G^{\prime} \nabla^{*} \lambda\right)(x)\right|, \\
& \left\|\zeta \nabla G^{\prime} \lambda\right\|_{\alpha}, \quad\left\|\zeta G^{\prime} \nabla^{*} \lambda\right\|_{\alpha}, \quad\left|\left(\Delta G^{\prime} \lambda\right)(x)\right| \\
& \leqq O(1)\left[\left(L^{j} \eta\right)^{2}, L^{j} \eta, L^{j} \eta,\left(L^{j} \eta\right)^{1-\alpha}\left(\|\zeta\|_{\alpha}+|\zeta|\right),\right. \\
& \left.\left(L^{j} \eta\right)^{1-\alpha}\left(\|\zeta\|_{\alpha}+|\zeta|\right), 1\right] \cdot e^{-\frac{1}{2} \delta_{0} d\left(y, y^{\prime}\right)}|\lambda|, \\
& x \in B^{j}(y) \text { or } \operatorname{supp} \zeta \subset B^{j}(y) \text {, } \\
& y \in \Lambda_{j}, \quad \operatorname{supp} \lambda \subset B^{j^{\prime}}\left(y^{\prime}\right), \quad y^{\prime} \in \Lambda_{j^{\prime}} .
\end{aligned}
$$

The random walk representation (2.50) is convergent in the norms defined by these inequalities. 
Let us consider now the operator $Q^{\prime} G^{\prime 2} Q^{\prime *}$ and its inverse $\left(Q^{\prime} G^{\prime 2} Q^{*}\right)^{-1}$. The inequalities (2.67) imply

$$
\begin{aligned}
&\left|\left(Q^{\prime} G^{\prime 2} Q^{\prime *}\right)(y, y)\right| \\
&=\left|\sum_{y^{\prime \prime} \in \mathfrak{B}}\left(Q^{\prime} G^{\prime} \Delta\left(y^{\prime \prime}\right) G^{\prime} Q^{\prime *}\right)(y, y)\right| \\
& \leqq \sum_{y^{\prime \prime} \in \mathfrak{B}} O(1)\left(L^{j} \eta\right)^{2} e^{-\frac{1}{2} \delta_{0} d\left(y, y^{\prime \prime}\right)}\left(L^{\prime \prime} \eta\right)^{2}\left(L^{j^{\prime}} \eta\right)^{-d} e^{-\frac{1}{2} \delta_{0} d\left(y^{\prime \prime}, y^{\prime}\right)} \\
& \leqq O(1)\left(L^{j} \eta\right)^{4}\left(L^{\prime} \eta\right)^{-d} \sum_{y^{\prime \prime} \in \mathfrak{B}} e^{-\frac{1}{6} \delta_{0} d\left(y, y^{\prime \prime}\right)} L^{2\left(j^{\prime \prime}-j\right)} e^{-\frac{1}{3} \delta_{0} d\left(y, y^{\prime \prime}\right)} e^{-\frac{1}{3} \delta_{0} d\left(y^{\prime \prime}, y^{\prime}\right)} \\
& \leqq O(1)\left(L^{j} \eta\right)^{4}\left(L^{\prime} \eta\right)^{-d} \sum_{y^{\prime \prime} \in \mathfrak{B}} e^{-\frac{1}{6} \delta_{0} R M \max \left\{\left|j^{\prime \prime}-j\right|-1,0\right\}} L^{L^{\left(j^{\prime \prime}-j\right)}} \\
& \cdot e^{-\frac{1}{3} \delta_{0} d\left(y, y^{\prime \prime}\right)} e^{-\frac{1}{3} \delta_{0} d\left(y^{\prime \prime}, y^{\prime}\right)} \leqq O(1)\left(L^{j} \eta\right)^{4}\left(L^{j^{\prime}} \eta\right)^{-d} e^{-\frac{1}{4} \delta_{0} d\left(y, y^{\prime}\right)},
\end{aligned}
$$

where we have used the inequalities (2.60), (2.63) of Lemma 1.

Let us notice that the choice of powers $\left(L^{j} \eta\right)^{4}$ and $\left(L^{j^{\prime}} \eta\right)^{-d}$ in the inequality above is purely conventional and we may change it into any other admissible choice, for example $\left(L^{j} \eta\right)^{-d}$ and $\left(L^{j^{\prime}} \eta\right)^{4}$, using the exponential factor $e^{-1 / 4 \delta_{0} d\left(y, y^{\prime}\right)}$ and the estimate $(2.60)$.

The inequalities (2.67), (2.68) suggest that we should consider the operator $Q^{\prime} G^{\prime 2} Q^{\prime *}$ on a Hilbert space $L^{2}(\mathfrak{B})$ defined by the scalar product

$$
\left\langle\lambda, \lambda^{\prime}\right\rangle=\sum_{j=0}^{k} \sum_{y \in \Lambda_{j}}\left(L^{j} \eta\right)^{d} \lambda(y) \lambda^{\prime}(y) .
$$

It is easy to see from the bound (2.58) that $Q^{\prime} G^{\prime 2} Q^{\prime *}$ is a bounded operator on this space; we have to use only the bound (2.61).

Now we will consider the operator $\left(Q^{\prime} G^{\prime 2} Q^{\prime *}\right)^{-1}$. Of course the operator $Q^{\prime} G^{\prime 2} Q^{\prime *}$ is positive definite, so its inverse is well defined. We will construct it and investigate its properties using again a random walk expansion. Our considerations are analogous to Sect. 5 of [3], concerning unit lattice operators. If we have one scale, i.e. $\Lambda_{k}=T_{1}^{(k)}$, then the operator is a unit lattice operator. The inverse operator is constructed by taking an approximate inverse and then solving an exact equation. The approximate inverse can be constructed by taking inverses of the localized operators $\left(Q^{\prime} G^{\prime 2} Q^{\prime *}\right) \Gamma_{\square}$ and glueing them together by the decomposition of unity $\left\{h_{\square}\right\}$. We change this prescription a little bit; we take a second cube $\tilde{\square}$ containing $\square$ in the middle and of the size $4 M$ and we take an inverse of the operator $\left(Q^{\prime} G^{\prime}(\tilde{\square})^{2} Q^{*}\right) \Gamma_{\square}$ instead of $\left(Q^{\prime} G^{\prime 2} Q^{*}\right) \Gamma_{\square}$. Let us define

$$
C_{\square}=\left(\left(Q^{\prime} G^{\prime}(\tilde{\square})^{2} Q^{*}\right) \Gamma_{\square}\right)^{-1}, \quad C=\sum_{\square \in \mathscr{D}} h_{\square} C_{\square} h_{\square} .
$$

At first let us find bounds on $C_{\square}$. We assume that either $\tilde{\square} \subset B^{j}\left(\Lambda_{j}\right)$, or it intersects $B^{j+1}\left(\Lambda_{j+1}\right)$ also. We have for $\omega$ defined on $\mathfrak{B} \cap \square$ (outside this set we put $\omega$ equal to 0$)$

$$
\begin{aligned}
& \left\langle\omega,\left(Q^{\prime} G^{\prime}(\tilde{\square})^{2} Q^{* *}\right) \Gamma_{\square} \omega\right\rangle \\
& \quad=\left\langle Q^{\prime *} \omega, Q_{j}^{\prime} G^{\prime \eta}(\tilde{\square})^{2} Q_{j}^{*} Q^{\prime *} \omega\right\rangle \\
& \quad=\left(L^{j} \eta\right)^{d+4}\left\langle Q^{\prime *} \omega, Q_{j}^{\prime} G^{\prime \xi}(\tilde{\square})^{2} Q_{j}^{*} Q^{\prime *} \omega\right\rangle, \quad \xi=L^{-j} .
\end{aligned}
$$


The scales of the operators were written explicitly and the last scalar product is on the unit scale. The operator $Q^{\prime \prime}$ is equal to $I$ on $\Lambda_{j} \cap \tilde{\square}$ and it is equal to $Q^{\prime}$ on $B\left(\Lambda_{j+1}\right) \cap \tilde{\square}=\Lambda$. Let us denote $\omega_{1}=Q^{\prime *} \omega$, hence $\omega_{1}$ is on the unit lattice and equals 0 outside $\square^{(j)}$. We have

$$
\left\langle\omega_{1}, Q_{j}^{\prime} G^{\prime \xi}(\tilde{\square})^{2} Q_{j}^{\prime *} \omega_{1}\right\rangle=\left\|G^{\prime \xi}(\tilde{\square}) Q_{j}^{* *} \omega_{1}\right\|^{2} .
$$

By Bessel's inequality the squared norm can be estimated from below by a sum of squares of coefficients in a decomposition of a vector with respect to an arbitrary orthonormal system. As the orthonormal system we take characteristic functions of unit blocks $\Delta=B^{j}(y) \subset \tilde{\square}$. We get

$$
\begin{aligned}
\left\|G^{\prime \xi}(\tilde{\square}) Q_{j}^{\prime *} \omega_{1}\right\|^{2} & \left.\geqq \sum_{\Delta} \mid\left\langle\Delta, G^{\prime \xi}(\tilde{\square}) Q_{j}^{\prime *} \omega_{1}\right\rangle\right)\left.\right|^{2} \\
& =\sum_{y \in \tilde{\square}(j)}\left|\left(Q_{j}^{\prime} G^{\prime \xi}(\tilde{\square}) Q_{j}^{\prime *} \frac{\omega_{1}}{\left\|\omega_{1}\right\|}\right)(y)\right|^{2}\left\|\omega_{1}\right\|^{2} \\
& \geqq\left|\sum_{y} \omega_{1}(y)\left(Q_{j}^{\prime} G^{\prime \xi}(\tilde{\square}) Q_{j}^{*} \frac{\omega_{1}}{\left\|\omega_{1}\right\|}\right)(y)\right|^{2} \\
& =\left|\left\langle\omega_{1}, Q_{j}^{\prime} G^{\prime \xi}(\tilde{\square}) Q_{j}^{\prime *} \omega_{1}\right\rangle\right|^{2} \frac{1}{\left\|\omega_{1}\right\|^{2}} .
\end{aligned}
$$

Next we consider the quadratic form $\left\langle\omega_{1}, Q_{j}^{\prime} G^{\prime \xi}(\tilde{\square}) Q_{j}^{\prime *} \omega_{1}\right\rangle$. Of course it is positive definite and to bound it from below we use Eq. (2.42):

$$
\begin{aligned}
& \left\langle\omega_{1}, Q_{j}^{\prime} G^{\prime \xi}(\tilde{\square}) Q_{j}^{\prime *} \omega_{1}\right\rangle \\
& \quad=\left\langle\omega_{1}, Q_{j}^{\prime} G_{j}^{\prime}(\tilde{\square}) Q_{j}^{\prime *} \omega_{1}\right\rangle+\left\langle\omega_{1},\left(Q_{j}^{\prime} G_{j}^{\prime}(\tilde{\square}) Q_{j}^{\prime *}\right) C_{\lambda}^{(j)}(\tilde{\square})\left(Q_{j}^{\prime} G_{j}^{\prime}(\tilde{\square}) Q_{j}^{\prime *}\right) \omega_{1}\right\rangle \\
& \quad \geqq\left\langle\omega_{1}, Q_{j}^{\prime} G_{j}^{\prime}(\tilde{\square}) Q_{j}^{*} \omega_{1}\right\rangle=\left\langle\omega_{1}, Q_{j}^{\prime} G_{j}^{\prime} Q_{j}^{\prime *} \omega_{1}\right\rangle+\left\langle\omega_{1}, Q_{j}^{\prime}\left(G_{j}^{\prime}(\tilde{\square})-G_{j}^{\prime}\right) Q_{j}^{\prime *} \omega_{1}\right\rangle \\
& \quad \geqq\left\langle\omega_{1}, Q_{j}^{\prime} G_{j}^{\prime} Q_{j}^{\prime *} \omega_{1}\right\rangle-O(1) e^{-\delta_{0} M}\left\|\omega_{1}\right\|^{2},
\end{aligned}
$$

where we have used the Theorem of [3], $G_{j}^{\prime}$ denotes the operator $\left(4^{L^{-j}}\right.$ $\left.+a_{j} Q_{j}^{\prime *} Q_{j}^{\prime}\right)^{-1}$ on the whole lattice $L^{-j} Z^{d}$. In [3] an explicit representation of this operator was found and from this representation we get the following Fourier representation of $Q_{j}^{\prime} G_{j}^{\prime} Q_{j}^{*}$ :

$$
\left(Q_{j}^{\prime} G_{j}^{\prime} Q_{j}^{\prime *}\right)\left(p^{\prime}\right)=\sum_{l} \frac{\left|u_{j}\left(p^{\prime}+l\right)\right|^{2} \Delta_{0}\left(p^{\prime}\right)}{\Delta\left(p^{\prime}+l\right)}\left(a_{j} \sum_{l} \frac{\left|u_{j}\left(p^{\prime}+l\right)\right|^{2} \Delta_{0}\left(p^{\prime}\right)}{\Delta\left(p^{\prime}+l\right)}+\Delta_{0}\left(p^{\prime}\right)\right)^{-1},
$$

$p^{\prime} \in[-\pi, \pi]^{d}, l$ was described in (2.45) [3]. From this representation and the bounds (2.50), (2.51) of that paper it follows that

$$
Q_{j}^{\prime} G_{j}^{\prime} Q_{j}^{*} \geqq 2 \gamma_{0} ;
$$

$\gamma_{0}$ is a positive, absolute constant $(a=1)$. For $M$ sufficiently large we obtain

$$
\left\langle\omega_{1}, Q_{j}^{\prime} G^{\prime \xi}(\tilde{\square}) Q_{j}^{\prime *} \omega_{1}\right\rangle \geqq \gamma_{0}\left\|\omega_{1}\right\|^{2} .
$$

The inequalities (2.73) and (2.77) together with the equalities (2.71) and (2.72) imply

$$
\left\langle\omega, Q^{\prime} G^{\prime \xi}(\tilde{\square})^{2} Q^{\prime *} \omega\right\rangle \geqq \gamma_{0}^{2}\left\|\omega_{1}\right\|^{2}=\gamma_{0}^{2}\left\|Q^{\prime \prime *} \omega\right\|^{2} \geqq \gamma_{0}^{2}\|\omega\|^{2} .
$$


Of course we have also a bound from above and an exponential decay of the kernel of $Q^{\prime} G^{\prime \xi}(\tilde{\square})^{2} Q^{*}$ with the decay rate $\delta_{0}$. Hence the operator $C_{\square}^{\xi}$ is bounded from above and below by absolute constants. We can use the theory developed in Sect. 5 [3] to conclude that it has an exponential decay with a decay rate $\delta_{1}$ depending on $\delta_{0}$ and the bound $\gamma_{0}$. Another way to prove it is to consider the operator $e^{\langle a, \cdot\rangle} Q^{\prime} G^{\prime \xi}(\tilde{\square})^{2} Q^{*} e^{-\langle a, \cdot\rangle}$, and to prove that it is almost equal to the operator without the exponential functions, the difference being of the order $O(|a|)$, for a small vector $a \in R^{d}$. This gives a bound for this operator almost the same as (2.78), which implies an exponential decay. Thus we have

$$
\left|C_{\square}^{\xi}\left(y, y^{\prime}\right)\right| \leqq O(1) e^{-\delta_{1}\left|y-y^{\prime}\right|}
$$

for $y, y^{\prime}$ belonging to $\mathfrak{B} \cap \square$ rescaled to unit scale. From (2.71) we obtain the following scaling law for the operators $C_{\square}$ :

hence

$$
C_{\square}\left(y, y^{\prime}\right)=\left(L^{j} \eta\right)^{-d-4} C_{\square}^{\xi}\left(\left(L^{j} \eta\right)^{-1} y,\left(L^{j} \eta\right)^{-1} y^{\prime}\right),
$$

$$
\left|C_{\square}\left(y, y^{\prime}\right)\right| \leqq O(1)\left(L^{j} \eta\right)^{-d-4} e^{-\delta_{1}\left(L^{j} \eta\right)^{-1}\left|y-y^{\prime}\right|}, \quad y, y^{\prime} \in \mathfrak{B} \cap \square .
$$

Now we will proceed in the same way as in Sect. 5, (5.12)-(5.17) [3]. We will show that $Q^{\prime} G^{\prime 2} Q^{*} C$ is a good approximation of identity. We have

$$
\begin{aligned}
Q^{\prime} G^{\prime 2} Q^{*} C= & \sum_{\square \in \mathscr{D}} Q^{\prime} G^{\prime 2} Q^{*} h_{\square} C_{\square} h_{\square}=\sum_{\square} h_{\square}\left(\square Q^{\prime} G^{\prime}(\tilde{\square})^{2} Q^{*} \square\right) C_{\square} h_{\square} \\
& -\sum_{\square}\left[h_{\square}, \square Q^{\prime} G^{\prime}(\tilde{\square})^{2} Q^{*} \square\right] C_{\square} h_{\square} \\
& -\sum_{\square} \square Q^{\prime}\left(G^{\prime}(\tilde{\square})^{2}-G^{\prime 2}\right) Q^{*} h_{\square} C_{\square} h_{\square} \\
& -\sum_{\square, \square^{\prime} \neq \square}(\square-1) h_{\square}^{2} Q^{\prime} G^{\prime 2} Q^{*} h_{\square} C_{\square} h_{\square} \\
= & I-\sum_{\square, \square^{\prime}} R_{\square, \square^{\prime}} C_{\square^{\prime}} h_{\square}=I-R,
\end{aligned}
$$

with an obvious definition of the operators $R_{\square, \square^{\prime}}$. We have to estimate the norm of $R$ in the space $L^{2}(\mathfrak{B})$. For example let us consider the operator $R_{\square, \square^{\prime}} C_{\square^{\prime}} h_{\square}$, with $\square \neq \square$ '. A kernel of this operator can be estimated as follows

$$
\begin{aligned}
& \left|\left(\square^{\prime}-1\right)(y) h_{\square}^{2}(y) \sum_{y^{\prime \prime} \in \operatorname{supp} h_{\square^{\prime}}}\left(L^{j^{\prime}} \eta\right)^{d}\left(Q^{\prime} G^{\prime 2} Q^{\prime *}\right)\left(y, y^{\prime \prime}\right) h_{\square^{\prime}}\left(y^{\prime \prime}\right) C_{\square^{\prime}}\left(y^{\prime \prime}, y^{\prime}\right) h_{\square}\left(y^{\prime}\right)\right| \\
& \quad \leqq O(1)\left(L^{j} \eta\right)^{4} \sum_{y^{\prime \prime} \in \operatorname{supp} h_{\square^{\prime}}} e^{-\frac{1}{2} \delta_{0} d\left(y, y^{\prime \prime}\right)} c_{1}\left(L^{j^{\prime}} \eta\right)^{-d-4} e^{-\delta_{1}\left(L^{j^{\prime}} \eta\right)^{-1}\left|y^{\prime \prime}-y^{\prime}\right|} \\
& \quad \leqq O(1)\left(L^{j} \eta\right)^{4}\left(L^{j^{\prime}} \eta\right)^{-4} e^{-\frac{1}{4} \delta_{0} d\left(y, \operatorname{supp} h_{\square^{\prime}}\right)-\delta_{1} d\left(y, y^{\prime}\right)\left(L^{j^{\prime}} \eta\right)^{-d}} \\
& \quad=O(1) e^{-\frac{1}{8} \delta_{0} M} L^{4\left(j-j^{\prime}\right)} e^{-\frac{1}{8} \delta_{0} R M \max \left\{\left|j-j^{\prime}\right|-1,0\right\}} e^{-\delta_{1} d\left(y, y^{\prime}\right)}\left(L^{j^{\prime}} \eta\right)^{-d} \\
& \quad \leqq O(1) e^{-\frac{1}{8} \delta_{0} M} e^{-\delta_{1} d\left(y, y^{\prime}\right)}\left(L^{j^{\prime}} \eta\right)^{-d}
\end{aligned}
$$

where we have used the fact that $y \notin \square^{\prime}$, and all the properties of the distance $d(\cdot, \cdot)$. From this inequality it follows that

$$
\left|R_{\square, \square}, C_{\square}, h_{\square}, \lambda\right| \leqq O(1) e^{-\frac{1}{8} \delta_{0} M} e^{-\frac{1}{2} \delta_{1} d\left(\square, \square^{\prime}\right)}|\lambda|,
$$


so for $M$ large enough the norm is small. Similar inequalities hold for kernels of the other operators forming $R$, for example the operator with $G^{\prime}(\tilde{\square})^{2}-G^{\prime 2}$ is small and an estimate has the factor $e^{-\delta_{0} M}$ because of the usual estimate of the type (1.12) [3] connected with a change of a domain. This estimate follows from the random walk representations $(2.50)$ for the operators $G^{\prime}, G^{\prime}(\tilde{\square})$. An estimate of the terms with the commutator is even simpler and gives a factor $O\left(M^{-1}\right)$. Now we can estimate the norm of $R$ either using the estimates of the type (2.84), or using an estimate of the kernel $R\left(y, y^{\prime}\right)$ of the operator $R$ following from all the partial estimates of the type (2.83). It can be written as

$$
\left|R\left(y, y^{\prime}\right)\right| \leqq O\left(M^{-1}\right) e^{-\delta_{1} d\left(y, y^{\prime}\right.}\left(L^{j^{\prime}} \eta\right)^{-d}, \quad y, y^{\prime} \in \mathfrak{B}, \quad y^{\prime} \in \Lambda_{j^{\prime}},
$$

and by Lemma 2.1 we get

Proposition 2.3. An inverse of the operator $Q^{\prime} G^{2} Q^{*}$ is given by the convergent expansion

$$
\begin{aligned}
\left(Q^{\prime} G^{\prime 2} Q^{*}\right)^{-1} & =C(I-R)^{-1}=\sum_{n=0}^{\infty} C R^{n} \\
& =\sum_{\omega} h_{\square_{0}} C_{\square_{0}} h_{\square 0} R_{\square_{1}, \square_{2}} C_{\square_{2}} h_{\square_{2}} \cdot \ldots \cdot R_{\square_{2 n-1}, \square_{2 n}} C_{\square_{2 n}} h_{\square_{2 n}},
\end{aligned}
$$

and it satisfies the estimate

$$
\begin{gathered}
\left|\left(Q^{\prime} G^{2} Q^{\prime *}\right)^{-1}(y, y)\right| \leqq O(1)\left(L^{j} \eta\right)^{-4}\left(L^{\prime} \eta\right)^{-d} e^{-\frac{1}{2} \delta_{1} d\left(y, y^{\prime}\right)} \\
y, y^{\prime} \in \mathfrak{B}, \quad y \in \Lambda_{j}, \quad y^{\prime} \in \Lambda_{j^{\prime}} .
\end{gathered}
$$

Finally let us consider the kernel of the operator $\partial P \partial^{*}$ appearing in $\partial R \partial^{*}$, $R=I-P$ given by (2.18). We have from Lemma 2.1, Proposition 2.2 and (2.87),

$$
\begin{aligned}
\left|\left(\partial P \partial^{*}\right)_{\mu v}\left(x, x^{\prime}\right)\right|= & \left|\left(\partial_{\mu} G^{\prime} Q^{*}\left(Q^{\prime} G^{\prime 2} Q^{\prime *}\right)^{-1} Q^{\prime} G^{\prime} \partial_{v}^{*}\right)\left(x, x^{\prime}\right)\right| \\
\leqq & O(1) \sum_{y_{1}, y_{2} \in \mathfrak{B}} L^{j} \eta e^{-\frac{1}{2} \delta_{0} d\left(y, y_{1}\right)}\left(L^{j_{1}} \eta\right)^{-4} e^{-\frac{1}{2} \delta_{1} d\left(y_{1}, y_{2}\right)}\left(L^{j_{2}} \eta\right)^{-d} \\
& \cdot e^{-\frac{1}{2} \delta_{0} d\left(y_{2}, y^{\prime}\right)} L^{j^{\prime}} \eta \leqq O(1)\left(L^{j} \eta\right)^{-2}\left(L^{\prime} \eta\right)^{-d} e^{-\delta_{2} d\left(y, y^{\prime}\right)},
\end{aligned}
$$

where $x \in B^{j}(y), x^{\prime} \in B^{j^{\prime}}(y), y \in \Lambda_{j}, y^{\prime} \in \Lambda_{j^{\prime}}$, and $\delta_{2}$ is determined by $\delta_{0}, \delta_{1}$. The choice of factors is again arbitrary and may be changed into any other admissible choice.

\section{Basic Operators}

Now we are ready to consider the operators $G$ and $\left(Q G Q^{*}\right)^{-1}$. We start with a construction of a random walk expansion analogous to the expansions (1.123) and (2.50). We use the partition $\mathscr{D}$ and the partition of unity $\left\{h_{\square}\right\}_{\square \in \mathscr{D}}$ as in (2.50). At first let us define operators $G_{\square}$ for the cubes $\square \in \mathscr{D}$. Let us take a cube connected with a $L^{j} \eta$-scale, i.e. either $\square C B^{j}\left(\Lambda_{j}\right)$, or it intersects also $B^{j+1}\left(\Lambda_{j+1}\right)$. A cube $\tilde{\square}$ is obtained from $\square$ by taking a sum of $4^{d}$ big blocks (of the size $M$ when rescaled to the proper scale) with distance to $\square$ equal to 0 . In the same way a cube $\tilde{\square}^{2}$ is formed, with $\tilde{\square}$ instead of $\square$ and built of $6^{d}$ big blocks, next a cube $\tilde{\square}^{3}$, and so on. We take the cube $\tilde{\square}^{3}$ and identify it with a torus, denoted by $T_{\square}$, imposing 
periodicity conditions. On this torus we define operators $R, \Delta_{a}$ as in (2.17), (2.19), but only two scales are present now. We define

$$
B^{j}(\Lambda)=\tilde{\square}^{2} \cap B^{j+1}\left(\Lambda_{j+1}\right),
$$

and we take $Q^{* *} a Q^{\prime}, Q^{*} a Q$ equal to $Q_{j+1}^{*} a_{j+1} Q_{j+1}^{\prime}, Q_{j+1}^{*} a Q_{j+1}$ on $B^{j}(\Lambda)$, and to $Q_{j}^{*} a_{j} Q_{j}^{\prime}, Q_{j}^{*} a Q_{j}$ on $T_{\square} \backslash B^{j}(\Lambda)$. Let us denote by $P_{\square}$ the projection operator in $(2.17)$ defined by the above $Q^{\prime *} a Q^{\prime}$, and

$$
G_{\square}=\left(\Delta-\partial P_{\square} \partial^{*}+Q^{*} a Q\right)^{-1} .
$$

Both operators are defined on the torus $T_{\square}$. We form an approximation of $G$ taking as usual

$$
G_{0}=\sum_{\square \in \mathscr{D}} h_{\square} G_{\square} h_{\square}
$$

Using the formulas (1.126)-(1.128), we get

$$
\Delta_{a} G_{0}=I-\sum_{\square, \square^{\prime} \in \mathscr{D}} K_{\square, \square^{\prime}} G_{\square^{\prime}} h_{\square^{\prime}}=I-R,
$$

where

$$
\begin{aligned}
\left(K_{\square, \square} A\right)_{\mu}(x)= & \sum_{b \in \text { st }(x)}\left(\partial h_{\square}\right)(b)\left(\partial A_{\mu}\right)(b)-\left(\Delta h_{\square}\right)(x) A_{\mu}(x) \\
& +a\left(L^{j} \eta\right)^{-2}\left(S_{j}^{*}\left(\partial h_{\square}\right) Q_{j} A\right)_{\mu}(x)-a\left(L^{j} \eta\right)^{-2}\left(Q_{j}^{*} S_{j}\left(\partial h_{\square}\right) A\right)_{\mu}(x) \\
& +\left(\zeta_{\square}\left(\partial P \partial^{*}-\partial P_{\square} \partial^{*}\right) h_{\square} A\right)_{\mu}(x) \\
& +\left(\zeta_{\square} P_{\square, 1}\left(\partial h_{\square}\right) A\right)_{\mu}(x) .
\end{aligned}
$$

if $x \in B^{j}\left(\Lambda_{j}\right)$ (we replace the index $j$ above by $j+1$ if $x \in B^{j+1}\left(\Lambda_{j+1}\right)$ ),

$$
\left(K_{\square, \square}, A\right)_{\mu}(x)=\left(h_{\square}^{2}\left(1-\zeta_{\square}\right) \partial P \partial^{*} h_{\square}, A\right)_{\mu}(x)
$$

if $\square \neq \square^{\prime}$. The operators $S_{j}, P_{\square, 1}\left(\partial h_{\square}\right)=\left[\partial P_{\square} \partial^{*}, h_{\square}\right]$ were defined in (1.120). The function $\zeta_{\square}$ is of the same type as $h_{\square}$, but it is equal to 1 on a cube containing $\square$ and with a boundary having the distance $1 / 3 M$ to the boundary of $\square$, and it is equal to 0 outside a similar cube with $1 / 3 M$ replaced by $2 / 3 M$.

To estimate the operators $K_{\square, \square}, G_{\square}, h_{\square}$, and $R$ we have to understand properties of the operator $G_{\square}$. We may assume that $B^{j}(\Lambda)=\tilde{\square}^{2} \cap B^{j+1}\left(\Lambda_{j+1}\right) \neq \emptyset$, because otherwise the operator $G_{\square}$ is simply equal to the operator $G_{j}$ on the torus $T_{\square}$. These operators were thoroughly investigated in paper [4], and their properties described in Proposition 1.2. We will prove that in the general case the operator $G_{\square}$ has the same properties. Unfortunately the situation is more complicated now because of the presence of the two scales. To prove these properties we will have to modify some arguments, as we had to in the case of operators $G^{\prime}(\square)$. At first we rescale $G_{\square}$ from $\eta$-lattice to $L^{-j}$-lattice. Of course we have

$$
G_{\square}^{\eta}\left(L^{j} \eta b, L^{j} \eta b^{\prime}\right)=\left(L^{j} \eta\right)^{-d+2} G_{\square}^{\xi}\left(b, b^{\prime}\right), \quad b, b^{\prime} \in T_{\square} \subset L^{-j} Z^{d},
$$

and correspondingly for derivatives of $G_{\square}$. This follows from the scaling laws for the operators defining $G_{\square}$ that $G_{\square}^{\xi}$ is defined by the same formula (2.90) with $\eta$ 
replaced by $\xi=L^{-j}$. For simplicity of notation let us omit $\xi$ and $\square$ in the formulas below.

To investigate the operator $G$ we will express it in terms of known operators in a way similar to $(2.41),(2.42)$. Thus we have

$$
\begin{aligned}
e^{\frac{1}{2}\langle J, G J\rangle}= & Z^{-1} \int d A \exp \left[-\frac{1}{2}\langle Q A, a Q A\rangle-\frac{1}{2}\left\langle A,\left(\Delta-\partial P \partial^{*}\right) A\right\rangle+\langle A, J\rangle\right] \\
= & Z^{-1} \int d A \exp \left[-\frac{1}{2}\langle Q A, a Q A\rangle-\frac{1}{2}\langle A, \Delta A\rangle+\langle A, J\rangle\right] \\
& \left(Z^{\prime-1} \int d \lambda \delta\left(Q^{\prime} \lambda\right) e^{\left.-\frac{1}{2}\left\|\partial^{*} A-\Delta \lambda\right\|^{2}\right)^{-1}}\right. \\
& \int d \omega \uparrow_{\Lambda} \delta\left(Q_{1}^{\prime} \omega\right) \prod_{y \in \Lambda^{\prime}} \delta_{A x(y)}\left(Q_{j} A+\partial_{1} \omega\right) Z_{j}^{\prime-1} \int d \lambda^{\prime} \delta\left(Q_{j}^{\prime} \lambda^{\prime}\right) e^{-\frac{1}{2}\left\|\partial^{*} A-\Delta \lambda^{\prime}\right\|^{2}} \\
& \cdot \frac{\int d \omega^{\prime} \uparrow_{\Lambda} \delta\left(Q_{1}^{\prime} \omega^{\prime}\right) \prod_{y \in \Lambda^{\prime}} \delta_{A x(y)}\left(Q_{j} A+\partial_{1} \omega^{\prime}\right) Z_{j}^{\prime-1} \int d \lambda^{\prime} \delta\left(Q_{j}^{\prime} \lambda^{\prime}\right) e^{-\frac{1}{2}\left\|\partial^{*} A-\Delta \lambda^{\prime}\right\|^{2}},}{}
\end{aligned}
$$

where we have used the identity (1.27) valid for this operator $P$ also. The configurations $\omega, \omega^{\prime}$ are defined on $\Lambda$ and from the definition of $\delta_{A x}$ we have

$$
\int d \omega^{\prime} \uparrow_{\Lambda} \delta\left(Q_{1}^{\prime} \omega^{\prime}\right) \prod_{y \in \Lambda^{\prime}} \delta_{A x(y)}\left(Q_{j} A+\partial_{1} \omega^{\prime}\right)=1 .
$$

Such an identity was used already in the change of gauge formula (1.23). The quotient in the last line of (2.95) was introduced to change the gauge fixing term in the integral by the Faddeev-Popov procedure. Let us apply (2.96) and (1.27) to the denominator. It defines the gauge fixing term introduced in Sect. C of [4], with blocks uniformly of order $j$. Let us denote the corresponding operator by $P_{j}$. We have

$$
\begin{aligned}
e^{\frac{1}{2}\langle J, G J\rangle} & \\
= & \int d \omega \uparrow_{\Lambda} \delta\left(Q_{1}^{\prime} \omega\right) Z^{-1} \int d A \exp \left[-\frac{1}{2} a \sum_{b \in \Lambda^{c}}\left|\left(Q_{j} A\right)(b)\right|^{2}-\frac{1}{2} a L^{d-2} \sum_{c \in \Lambda^{\prime}}\left|\left(Q_{j+1} A\right)(c)\right|^{2}\right. \\
& \left.-\frac{1}{2}\|\partial A\|^{2}-\frac{1}{2}\left\|\left(I-P_{j}\right) \partial^{*} A\right\|^{2}+\langle A, J\rangle\right] \\
& \cdot \prod_{y \in \Lambda^{\prime}} \delta_{A x(y)}\left(Q_{j} A+\partial_{1} \omega\right) Z_{j}^{\prime-1} \int d \lambda^{\prime} \delta\left(Q_{j}^{\prime} \lambda\right) e^{-\frac{1}{2}\left\|\partial^{*} A-\Delta \lambda^{\prime}\right\|^{2}} \\
& \cdot\left(Z^{\prime-1} \int d \lambda \delta\left(Q^{\prime} \lambda\right) e^{-\frac{1}{2}\left\|\partial^{*} A-\Delta \lambda\right\|^{2}}\right)^{-1} .
\end{aligned}
$$

Our next step will be to make a gauge transformation in the integral $\int d A \ldots$ which will transform the functions $\delta_{A x(y)}\left(Q_{j} A+\partial_{1} \omega\right)$ into $\delta_{A x(y)}\left(Q_{j} A\right)$. We want to make a gauge transformation which does not change the quadratic form in the first exponential function above. At first let us find gauge transformations $\lambda$ which do not change the last term in the quadratic form, i.e. for which

$$
\left\|\left(I-P_{j}\right) \partial^{*} A^{\lambda}\right\|^{2}=\left\|\left(I-P_{j}\right)\left(\partial^{*} A-\Delta \lambda\right)\right\|^{2}=\left\|\left(I-P_{j}\right) \partial^{*} A\right\|^{2} .
$$

This equality has to be satisfied for all $A$, hence for $A=0$ we get the equation

$$
\left(I-P_{j}\right) \Delta \lambda=0, \text { or } \Delta \lambda=P_{j} \Delta \lambda \text {. }
$$

If this equation is satisfied, then the equality (2.98) holds for all $A$. The function $\Delta \lambda$ is orthogonal to constant functions, hence $P_{j} \Delta \lambda$ is given by (1.26) and we have

$$
\Delta \lambda=\Delta^{-1} Q_{j}^{*}\left(Q_{j}^{\prime} \Delta^{-2} Q_{j}^{\prime *}\right)^{-1} Q_{j}^{\prime} \Delta^{-1} \Delta \lambda
$$


We may assume also that $\lambda$ is orthogonal to constant functions because a constant component of $\lambda$ does not influence the gauge transformation defined by $\partial \lambda$, hence

$$
\lambda=\Delta^{-2} Q_{j}^{*}\left(Q_{j}^{\prime} \Delta^{-2} Q_{j}^{*}\right)^{-1} \mu, \quad \mu=Q_{j}^{\prime} \lambda
$$

Thus the gauge transformations satisfying (2.98) are parametrized by functions $\mu$ defined on the unit lattice and orthogonal to constant functions, and are given by the formula (2.101). The functions $\mu$ satisfy $\mu=Q_{j}^{\prime} \lambda$. Let us consider this formula more closely. It is easy to see that it defines an operator which is connected with the integral

$$
Z_{j}^{\prime-1} \int d \lambda^{\prime} \delta\left(Q_{j}^{\prime} \lambda^{\prime}-\mu\right) e^{-\frac{1}{2}\left\|\Delta \lambda^{\prime}\right\|^{2}}
$$

in the same way as the operator $H_{k}$ was connected with the integral (1.47). It gives a solution of the variational problem $\inf _{\lambda^{\prime}: Q_{j}^{\prime} \lambda^{\prime}=\mu} \frac{1}{2}\left\|\Delta \lambda^{\prime}\right\|^{2}$. Let us denote the operator in (2.101) by $H_{j}^{\prime}$, so $\lambda=H_{j}^{\prime} \mu$. From the momentum representation of (2.101) we may get easily that $H_{j}^{\prime}$ is a bounded operator with an exponential decay, the bound and decay rate depending on $d$ only.

Let us come back to our problem of finding gauge transformations leaving the quadratic form invariant. The transformations given by $\lambda=H_{j}^{\prime} \mu$ are the only ones leaving invariant the third and fourth terms in the quadratic form. For the averages in the first two terms we have

$$
\begin{gathered}
\left(Q_{j} A^{\lambda}\right)(b)=\left(Q_{j} A\right)(b)-\left(\left(Q_{j}^{\prime} \lambda\right)\left(b_{+}\right)-\left(Q_{j}^{\prime} \lambda\right)\left(b_{-}\right)\right)=\left(Q_{j} A\right)(b)-\left(\partial_{1} \mu\right)(b) \\
\left(Q_{j+1} A^{\lambda}\right)(c)=\left(Q_{j+1} A\right)(c)-L^{-1}\left(\left(Q_{j+1}^{\prime} \lambda\right)\left(c_{+}\right)-\left(Q_{j+1}^{\prime} \lambda\right)\left(c_{-}\right)\right) \\
=\left(Q_{j+1} A\right)(c)-\left(\partial^{L} Q_{1}^{\prime} \mu\right)(c),
\end{gathered}
$$

where the equality $Q_{j}^{\prime} \lambda=Q_{j}^{\prime} H_{j}^{\prime} \mu=\mu$ was used. Hence these terms are invariant if and only if $\partial_{1} \mu=0$ on $\Lambda^{c}$ and $\partial^{L} Q_{1}^{\prime} \mu=0$ on $\Lambda^{\prime}$. This is possible only if $\mu$ is constant on $\Lambda^{c}$ and $Q_{1}^{\prime} \mu$ is constant on $\Lambda^{\prime}$ and the constants are equal. But we have assumed that $\mu$ is orthogonal to constant functions, so the constant is equal to 0 and we have

$$
\mu=0 \text { on } \Lambda^{c}, \quad Q_{1}^{\prime} \mu=0 \text { on } \Lambda^{\prime} .
$$

This matches our needs exactly because the unit lattice gauge functions $\omega$ appearing in the integral (2.97) satisfy the above conditions. Let us make a gauge transformation in the integral $\int d A \ldots$ defined by the function $\lambda_{0}=H_{j}^{\prime} \omega, A \rightarrow A-\partial \lambda_{0}$ $=A-\partial H_{j}^{\prime} \omega$. This transformation does not change the measure $d A$ and in the first exponential only the term $\langle A, J\rangle$ is changed into $\left\langle A-\partial H_{j}^{\prime} \omega, J\right\rangle$. According to (2.103) the $\delta$-functions $\delta_{A x(y)}\left(Q_{j} A+\partial_{1} \omega\right)$ are changed into $\delta_{A x(y)}\left(Q_{j} A\right)$. In the first integral $\int d \lambda^{\prime} \ldots$ we get the expression $\partial^{*} A^{\lambda_{0}}-\Delta \lambda^{\prime}=\partial^{*} A-\Delta\left(\lambda^{\prime}+\lambda_{0}\right)$, and we make the transformation $\lambda^{\prime} \rightarrow \lambda^{\prime}-\lambda_{0}$. After the transformation we obtain the same integral with the $\delta$-functions $\delta\left(Q_{j}^{\prime} \lambda^{\prime}\right)$ replaced by $\delta\left(Q_{j}^{\prime} \lambda^{\prime}-Q_{j}^{\prime} \lambda_{0}\right)=\delta\left(Q_{j}^{\prime} \lambda^{\prime}-\omega\right)$. In the last integral in $\left(Z^{\prime-1} \int d \lambda \ldots\right)^{-1}$ we make the same transformations, only now in $\delta$-functions we get $Q^{\prime} \lambda-Q^{\prime} \lambda_{0}$, and $Q^{\prime} \lambda_{0}=Q_{j}^{\prime} \lambda_{0}=\omega=0$ on $\Lambda^{c}$, and $Q^{\prime} \lambda_{0}=Q_{j+1}^{\prime} \lambda_{0}$ $=Q_{1}^{\prime} \omega=0$ on $\Lambda^{\prime}$. Thus this integral is not changed by the transformation. Taking 
into account all these changes we get

$$
\begin{aligned}
e^{\frac{1}{2}\langle J, G J\rangle} & Z^{-1} \int d A \prod_{y \in \Lambda^{\prime}} \delta_{A x(y)}\left(Q_{j} A\right) \exp \left[-\frac{1}{2}\langle Q A, a Q A\rangle\right. \\
& \left.-\frac{1}{2}\left\langle A,\left(\Delta-\partial P_{j} \partial^{*}\right) A\right\rangle+\langle A, J\rangle\right] \\
& \cdot \int d \omega \uparrow_{\Lambda} \delta\left(Q_{1}^{\prime} \omega\right) Z_{j}^{\prime-1} \int d \lambda^{\prime} \delta\left(Q_{j}^{\prime} \lambda^{\prime}-\omega\right) e^{-\frac{1}{2}\left\|\partial^{*} A-\Delta \lambda^{\prime}\right\|^{2}} e^{-\left\langle\partial H_{j}^{\prime} \omega, J\right\rangle} \\
& \cdot\left(Z^{\prime-1} \int d \lambda \delta\left(Q^{\prime} \lambda\right) e^{-\frac{1}{2}\left\|\partial^{*} A-\Delta \lambda\right\|^{2}}\right)^{-1}
\end{aligned}
$$

Let us notice that $\int d \omega^{\prime} \uparrow_{\Lambda} \delta\left(Q_{1}^{\prime} \omega^{\prime}\right) \delta\left(Q_{j}^{\prime} \lambda^{\prime}-\omega^{\prime}\right)=\delta\left(Q^{\prime} \lambda\right)$, and we use this formula in the last integral above. The integrals over $\omega$ 's and $\lambda$ 's can be written as

$$
\begin{aligned}
& \int d \omega \uparrow_{\Lambda} \delta\left(Q_{1}^{\prime} \omega\right) \int d \lambda^{\prime} \delta\left(Q_{j}^{\prime} \lambda^{\prime}-\omega\right) e^{-\frac{1}{2}\left\|\Delta \lambda^{\prime}\right\|^{2}+\left\langle\Delta \lambda^{\prime}, \partial^{*} A\right\rangle} e^{-\left\langle\partial H_{j}^{\prime} \omega, J\right\rangle} \\
& \\
& \cdot\left(\int d \omega^{\prime} \uparrow_{\Lambda} \delta\left(Q_{1}^{\prime} \omega^{\prime}\right) \int d \lambda \delta\left(Q_{j}^{\prime} \lambda-\omega^{\prime}\right) e^{-\frac{1}{2}\|\Delta \lambda\|^{2}+\left\langle\Delta \lambda, \partial^{*} A\right\rangle}\right)^{-1} \\
&=\int d \omega \uparrow_{\Lambda} \delta\left(Q_{1}^{\prime} \omega\right) e^{-\frac{1}{2}\left\|\Delta H_{j}^{\prime} \omega\right\|^{2}} e^{\left\langle\Delta H_{j}^{\prime} \omega, \partial^{*} A\right\rangle-\left\langle\partial H_{j}^{\prime} \omega, J\right\rangle} \\
& \cdot \int d \lambda^{\prime} \delta\left(Q_{j}^{\prime} \lambda\right) e^{-\frac{1}{2}\left\|\Delta \lambda^{\prime}\right\|^{2}+\left\langle\Delta \lambda^{\prime}, \partial^{*} A\right\rangle} \\
& \cdot\left(\int d \omega^{\prime} \uparrow_{\Lambda} \delta\left(Q_{1}^{\prime} \omega^{\prime}\right) e^{-\frac{1}{2}\left\|\Delta H_{j}^{\prime} \omega^{\prime}\right\|^{2}} e^{\left\langle\Delta H_{j}^{\prime} \omega^{\prime}, \partial^{*} A\right\rangle} \int d \lambda \delta\left(Q_{j}^{\prime} \lambda\right) e^{-\frac{1}{2}\|\Delta \lambda\|^{2}+\left\langle\Delta \lambda, \partial^{*} A\right\rangle}\right)^{-1} \\
&= \int d \omega \uparrow_{\Lambda} \delta\left(Q_{1}^{\prime} \omega\right) e^{-\frac{1}{2}\left\langle\omega, \Delta_{j}^{\prime} \omega\right\rangle} e^{\left\langle\omega, H_{j}^{\prime} \Delta \hat{o}^{*} A-H_{j}^{*} \partial^{*} J\right\rangle} \\
& \cdot\left(\int d \omega^{\prime} \uparrow_{\Lambda} \delta\left(Q_{1}^{\prime} \omega^{\prime}\right) e^{-\frac{1}{2}\left\langle\omega^{\prime}, \Delta_{j}^{\prime} \omega^{\prime}\right\rangle} e^{\left\langle\omega^{\prime}, H_{j}^{*} \Delta \partial^{*} A\right\rangle}\right)^{-1},
\end{aligned}
$$

where the second equality was obtained by the translation $\lambda^{\prime} \rightarrow \lambda^{\prime}+H_{j}^{\prime} \omega$, and the same translation in $\lambda$, and the operator $\Delta_{j}^{\prime}$ was defined by the second equality,

$$
\Delta_{j}^{\prime}=H_{j}^{\prime *} \Delta^{2} H_{j}^{\prime} \text {. }
$$

In momentum representation on the unit lattice the operator $\Delta_{j}^{\prime}$ is represented as the multiplication operator by the function

$$
\Delta_{j}^{\prime}\left(p^{\prime}\right)=\Delta_{0}^{2}\left(p^{\prime}\right)\left(\sum_{l} \frac{\left|u_{j}\left(p^{\prime}+l\right)\right|^{2} \Delta_{0}^{2}\left(p^{\prime}\right)}{\Delta^{2}\left(p^{\prime}+l\right)}\right)^{-1} .
$$

From this we get an exponential decay of $\Delta_{j}^{\prime}\left(y-y^{\prime}\right)$ and the bound

$$
c_{0}\left\|\Delta_{0} \omega\right\|^{2} \leqq\left\langle\omega, \Delta_{j}^{\prime} \omega\right\rangle \leqq c_{1}\left\|\Delta_{0} \omega\right\|^{2} \leqq \gamma_{1}\|\omega\|^{2} .
$$

For $\omega$ satisfying $Q_{1}^{\prime} \omega=0$ the quadratic form $\left\|\Delta_{0} \omega\right\|^{2}=\left\|\Delta_{0} \omega+a Q_{1}^{*} Q_{1}^{\prime} \omega\right\|^{2}$ is bounded from below by $\gamma_{0}^{\prime 2}\|\omega\|^{2}$ because the operator $\Delta_{0}+a Q_{1}^{\prime *} Q_{1}^{\prime}$ is bounded from below by $\gamma_{0}^{\prime}>0$ (in fact we may get $\gamma_{0}^{\prime}=\pi^{2} / L^{2}$ ). Hence

$$
\gamma_{0}\|\omega\|^{2} \leqq\left\langle\omega, \Delta_{j}^{\prime} \omega\right\rangle \leqq \gamma_{1}\|\omega\|^{2} \text { for } \omega: Q_{1}^{\prime} \omega=0
$$

with positive constants $\gamma_{0}, \gamma_{1}$ dependent on $d$ and $L$ only. From the theorem on unit lattice operators in [3] it follows that a covariance $C_{\Lambda}^{(j)}$ of the last Gaussian integrals in (2.106) is a bounded operator with an exponential decay independent of $j$ and $\Lambda$. Calculating these integrals we get

$$
\begin{aligned}
& \exp \left[-\left\langle H_{j}^{\prime *} \Delta \partial^{*} A, C_{\Lambda}^{\prime(j)} H_{j}^{\prime *} \partial^{*} J\right\rangle+\frac{1}{2}\left\langle H_{j}^{\prime *} \partial^{*} J, C_{\Lambda}^{\prime(j)} H_{j}^{* *} \partial^{*} J\right\rangle\right] \\
& \quad=\exp \left[-\left\langle A, \partial \Delta H_{j}^{\prime} C_{\Lambda}^{\prime(j)} H_{j}^{\prime *} \partial^{*} J\right\rangle+\frac{1}{2}\left\langle J, \partial H_{j}^{\prime} C_{\Lambda}^{\prime(j)} H_{j}^{\prime *} \partial^{*} J\right\rangle\right] .
\end{aligned}
$$


This implies further

$$
\begin{aligned}
e^{\frac{1}{2}\langle J, G J\rangle}= & e^{\frac{1}{2}\left\langle J, \partial H_{j}^{\prime} C_{A}^{\prime(j)} H_{j}^{* *} \partial^{*} J\right\rangle} Z^{-1} Z_{j}^{\prime-1} Z^{\prime} \\
& \cdot \int d B \exp \left[-\frac{1}{2} a \sum_{b \in A^{c}}|B(b)|^{2}-\frac{1}{2} a L^{d-2} \sum_{c \in A^{\prime}}\left|\left(Q_{1} B\right)(c)\right|^{2}\right] \prod_{y \in A^{\prime}} \delta_{A x(y)}(B) \\
& \cdot \int d A \delta\left(Q_{j} A-B\right) \exp \left[-\frac{1}{2}\left\langle A,\left(\Delta-\partial P_{j} \partial^{*}\right) A\right\rangle\right. \\
& \left.+\left\langle A, J-\partial \Delta H_{j}^{\prime} C_{A}^{\prime(j)} H_{j}^{\prime *} \partial^{*} J\right\rangle\right] .
\end{aligned}
$$

In the last integral above we make the translation $A \rightarrow A+H_{j} B$. The operator $H_{j}$ may be different from the operator defined in Sect. D of [4], because there is the exponential gauge fixing term instead of the $\delta$-function, but we will prove later that they are equal.

The translation gives the factor

$$
\exp \left[-\frac{1}{2}\left\langle H_{j} B,\left(\Delta-\partial P_{j} \partial^{*}\right) H_{j} B\right\rangle+\left\langle H_{j} B, J-\partial \Delta H_{j}^{\prime} C_{A}^{\prime(j)} H_{j}^{*} \partial^{*} J\right\rangle\right]
$$

and separates the integral over $A$ :

$$
\int d A \delta\left(Q_{j} A\right) \exp \left[-\frac{1}{2}\left\langle A,\left(\Delta-\partial P_{j} \partial^{*}\right) A\right\rangle+\langle A, J-\ldots\rangle\right] .
$$

Let us denote a covariance of this Gaussian integral by $\widetilde{G}_{j}$, then the integral is equal to

Let us notice also that

$$
\tilde{Z}_{j} \exp \left[\frac{1}{2}\left\langle J-\ldots, \tilde{G}_{j}(J-\ldots)\right\rangle\right] .
$$

$$
\begin{aligned}
& \exp \left[-\frac{1}{2}\left\langle H_{j} B,\left(\Delta-\partial P_{j} \partial^{*}\right) H_{j} B\right\rangle\right] \\
& \quad=\tilde{Z}_{j}^{-1} \int d A \delta\left(Q_{j} A-B\right) \exp \left[-\frac{1}{2}\left\langle A,\left(\Delta-\partial P_{j} \partial^{*}\right) A\right\rangle\right],
\end{aligned}
$$

and that in the last integral we may replace the exponential gauge fixing term by the $\delta$-function $\delta_{R}\left(R \partial^{*} A\right)$ using the Faddeev-Popov procedure. Then this integral gives the factor

$$
\exp \left[-\frac{1}{2}\left\|\partial H_{j} B\right\|^{2}\right]
$$

by (1.47) and (1.64). Thus both factors are equal and in fact the quadratic forms are equal to $\left\langle B, \Delta_{j} B\right\rangle$ given by (1.66) and satisfying (1.67):

$$
\gamma_{0}\left\|\partial_{1} B\right\|^{2} \leqq\left\langle B, \Delta_{j} B\right\rangle \leqq \gamma_{1}\left\|\partial_{1} B\right\|^{2} .
$$

These calculations give

$$
\begin{aligned}
e^{\frac{1}{2}\langle J, G J\rangle}= & \exp \left[\frac{1}{2}\left\langle J, \partial H_{j}^{\prime} C_{A}^{(j)} H_{j}^{*} \partial^{*} J\right\rangle\right. \\
& \left.+\frac{1}{2}\left\langle J-\partial \Delta H_{j}^{\prime} C_{A}^{(j)} H_{j}^{*} \partial^{*} J, \widetilde{G}_{j}\left(J-\partial \Delta H_{j}^{\prime} C_{A}^{(j)} H_{j}^{\prime *} \partial^{*} J\right)\right\rangle\right] \\
& \cdot Z^{\prime \prime}-1 \int d B \prod_{y \in A^{\prime}} \delta_{A x(y)}(B) \exp \left[-\frac{1}{2}\left\langle Q^{\prime \prime} B, a Q^{\prime \prime} B\right\rangle-\frac{1}{2}\left\langle B, \Delta_{j} B\right\rangle\right. \\
& \left.+\left\langle B, H_{j}^{*}\left(J-\partial \Delta H_{j}^{\prime} C_{A}^{(j)} H_{j}^{\prime *} \partial^{*} J\right)\right\rangle\right],
\end{aligned}
$$

where $\frac{1}{2}\left\langle Q^{\prime \prime} B, a Q^{\prime \prime} B\right\rangle$ is equal to the quadratic form in $B$ in (2.112). Now let us consider the integral above. It is a Gaussian integral defined by the quadratic form 
(we take $a=1$ )

$$
\left\|B \uparrow_{\Lambda^{c}}\right\|^{2}+L^{-2}\left\|\left(Q_{1} B\right) \uparrow_{\Lambda^{\prime}}\right\|^{2}+\left\langle B, \Delta_{j} B\right\rangle
$$

on the configurations $B$ satisfying

$$
B(b)=0 \text { for } b \subset \Gamma_{y, x}, \quad x \in B(y), \quad y \in \Lambda^{\prime} .
$$

The form is bounded from above, and bounded from below by $\left\|B \uparrow_{A^{c}}\right\|^{2}$. There is also the bound (2.118), so let us consider the form

$$
\left\|B \uparrow_{\Lambda^{c}}\right\|^{2}+L^{-2}\left\|\left(Q_{1} B\right) \uparrow_{\Lambda^{\prime}}\right\|^{2}+\gamma_{0}\left\|\partial_{1} B\right\|^{2} .
$$

We will prove that it is bounded from below by $\gamma_{0}^{\prime \prime}\|B\|^{2}$ on the configurations $B$ satisfying (2.121). Let us consider at first the bonds $b \subset B(y), y \in \Lambda^{\prime}$. For $b=\left\langle x, x+e_{2}\right\rangle$, we have

$$
\begin{aligned}
B\left(x, x+e_{2}\right)= & \left(B\left(x, x+e_{2}\right)-B\left(x-e_{1}, x-e_{1}+e_{2}\right)\right) \\
& +\ldots+\left(B\left(\left(y_{1}+1, x_{2}, \ldots, x_{d}\right),\left(y_{1}+1, x_{2}+1, \ldots, x_{d}\right)\right)\right. \\
& \left.-B\left(\left(y_{1}, x_{2}, \ldots, x_{d}\right),\left(y_{1}, x_{2}+1, \ldots, x_{d}\right)\right)\right)=\sum\left(\partial_{1} B\right)\left(p\left(x^{\prime}\right)\right),
\end{aligned}
$$

the sum is over the plaquettes $p\left(x^{\prime}\right)$ determined by the points $x^{\prime} \in\left[\left(y_{1}\right.\right.$, $\left.\left.x_{2}, \ldots, x_{d}\right), x\right]$ and vectors $e_{1}, e_{2}$, so

$$
\left|B\left(x, x+e_{2}\right)\right|^{2} \leqq\left|x_{1}-y_{1}\right| \sum\left|\left(\partial_{1} B\right)\left(p\left(x^{\prime}\right)\right)\right|^{2} .
$$

If we take a bond $b=\left\langle x, x+e_{3}\right\rangle$, then in the above bound we will have to include the points

$$
x^{\prime} \in\left[\left(y_{1}, y_{2}, x_{3}, \ldots, x_{d}\right),\left(y_{1}, x_{2}, x_{3}, \ldots, x_{d}\right)\right]
$$

also. For arbitrary $j, 2 \leqq j \leqq d$, we have

$$
\left|B\left(x, x+e_{j}\right)\right|^{2} \leqq\left(\left|x_{1}-y_{1}\right|+\ldots+\left|x_{j-1}-y_{j-1}\right|\right) \sum\left|\left(\partial_{1} B\right)\left(p\left(x^{\prime}\right)\right)\right|^{2},
$$

the sum is over

$$
x^{\prime} \in\left[\left(y_{1}, \ldots, y_{j-1}, x_{j}, \ldots, x_{d}\right),\left(y_{1}, \ldots, x_{j-1}, x_{j}, \ldots, x_{d}\right)\left[\cup \ldots \cup\left[\left(y_{1}, x_{2}, \ldots, x_{d}\right), x\right]\right.\right.
$$

and the plaquettes $p\left(x^{\prime}\right)$ are parallel to $\left(e_{j-1}, e_{j}\right), \ldots,\left(e_{1}, e_{j}\right)$. We sum the above inequalities over $x$ and $j=2, \ldots, d$. The sum over $j$ involves disjoint sets of plaquettes on the right-hand side, so to get a constant in the inequality we have to consider the sum over $x$. The worst situation is for $j=d$ and it is easy to see that

$$
\sum_{x:\left\langle x, x+e_{d}\right\rangle \subset B(y)}\left|B\left(x, x+e_{d}\right)\right|^{2} \leqq(d-1)(L-1)^{2} L^{d-2} \Sigma_{d}^{\prime}\left|\left(\partial_{1} B\right)(p)\right|^{2},
$$

hence

$$
\sum_{b \subset B(y)}|B(b)|^{2} \leqq d L^{d} \sum_{p \subset B(y)}\left|\left(\partial_{1} B\right)(p)\right|^{2} .
$$

Next let us consider bonds $b \in B(c)=\left\{b: b_{-} \in B\left(c_{-}\right), b_{+} \in B\left(c_{+}\right)\right\}$for some $c \in \Lambda^{\prime}$. Let $c=\left\langle y, y+L e_{\mu}\right\rangle$ and $\Delta^{\prime}, \Delta^{\prime \prime}$ denote the intersection of the planes $x_{\mu}=c_{+, \mu}-1$ $=y_{\mu}+(L-1), x_{\mu}=c_{+, \mu}=y_{\mu}+L$ with the blocks $B\left(c_{-}\right), B\left(c_{+}\right)$correspondingly, as indicated in the figure below. 


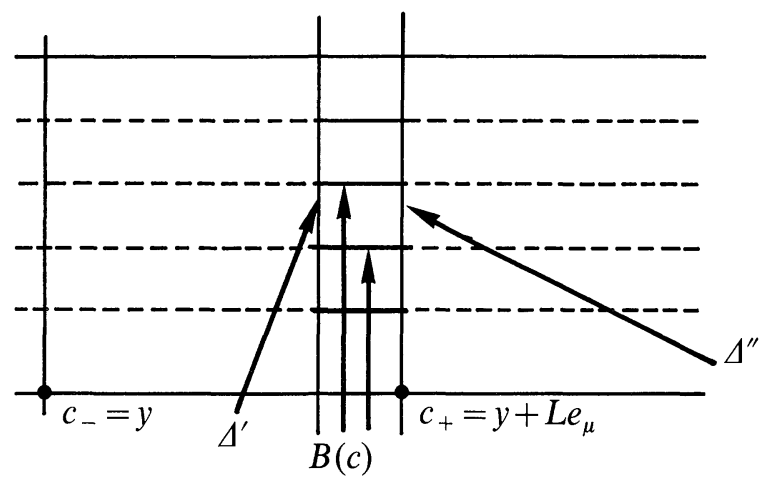

We identify $B(b)=B_{\mu}(x)$ for $b=\left\langle x, x+e_{\mu}\right\rangle$, and we have

$$
\begin{aligned}
& \sum_{p \subset B(c)}\left|\left(\partial_{1} B\right)(p)\right|^{2} \geqq \frac{1}{3} \sum_{b \subset \Delta^{\prime}}\left|\left(\partial B_{\mu}\right)(b)\right|^{2}-\sum_{b \subset \Delta^{\prime}}|B(b)|^{2}-\sum_{b \subset \Delta^{\prime \prime}}|B(b)|^{2}, \\
&\left|\left(Q_{1} B\right)(c)\right|^{2}=\left|\sum_{x \in B(c-)} L^{-(d+1)} B\left(\left[x, x+L e_{\mu}\right]\right)\right|^{2}=\mid L^{-d} \sum_{x \in B(y) \backslash \Delta^{\prime}} L^{-1}\left(x_{\mu}-y_{\mu}+1\right) B_{\mu}(x) \\
&+L^{-d} \sum_{x \in \Delta^{\prime}} B_{\mu}(x)+\left.L^{-d} \sum_{x \in B\left(y+L e_{\mu}\right)} L^{-1}\left(2 L-1+y_{\mu}-x_{\mu}\right) B_{\mu}(x)\right|^{2} \\
& \leqq \frac{1}{3}\left|\sum_{x \in \Delta^{\prime}} L^{-d} B_{\mu}(x)\right|^{2}-L^{-d} \sum_{\left\langle x, x+e_{\mu}\right\rangle \subset B\left(c_{-}\right)}\left|B_{\mu}(x)\right|^{2} \\
&-L^{-d} \sum_{\left\langle x, x+e_{\mu}\right\rangle \subset B\left(c_{+}\right)}\left|B_{\mu}(x)\right|^{2} .
\end{aligned}
$$

From these two inequalities we obtain

$$
\begin{aligned}
& L^{-d} \sum_{p \subset B(c)}\left|\left(\partial_{1} B\right)(p)\right|^{2}+\left|\left(Q_{1} B\right)(c)\right|^{2} \geqq \frac{1}{3}\left(L^{-d} \sum_{b \subset \Delta^{\prime}}\left|\left(\partial B_{\mu}\right)(b)\right|^{2}+L^{-2}\left|\sum_{x \in \Delta^{\prime}} L^{-(d-1)} B_{\mu}(x)\right|^{2}\right) \\
& -L^{-d} \sum_{b \subset B\left(c_{-}\right)}|B(b)|^{2}-L^{-d} \sum_{b \subset B\left(c_{+}\right)}|B(b)|^{2} .
\end{aligned}
$$

The terms in parentheses on the right-hand side can be written as $L^{-2}\left\langle B,\left(\Delta_{\Delta^{\prime}}^{L-1, N}\right.\right.$ $\left.\left.+Q_{\Delta^{\prime}}^{\prime *} Q_{\Delta^{\prime}}^{\prime}\right) B\right\rangle$, where the operators are defined on a $d-1$-dimensional lattice. This quadratic form is bounded from below by $L^{-d-1} \sum_{x \in \Delta^{\prime}}\left|B_{\mu}(x)\right|^{2}$, hence

$$
\begin{aligned}
& L^{-d} \sum_{p \subset B(c)}\left|\left(\partial_{1} B\right)(p)\right|^{2}+\left|\left(Q_{1} B\right)(c)\right|^{2} \geqq \frac{1}{3} L^{-d-1} \sum_{b \in B(c)}|B(b)|^{2} \\
& \quad-L^{-d} \sum_{b \subset B\left(c_{-}\right)}|B(b)|^{2}-L^{-d} \sum_{b \subset B\left(c_{+}\right)}|B(b)|^{2} .
\end{aligned}
$$

The inequalities (2.123) and (2.127) imply many other inequalities. One of them is formulated in

Lemma 2.4. Let a set $\Lambda \subset Z^{d}$ be a sum of blocks, $\Lambda=B\left(\Lambda^{\prime}\right)$. We denote by $\Lambda$ also a set of bonds $b$ such that at least one of the end-points $b_{-}, b_{+}$belongs to $\Lambda$. Let $B$ be $a$ configuration defined on $\Lambda$ and satisfying the condition $(2.121): B\left(\Gamma_{y, x}\right)=0$ for $x \in B(y), y \in \Lambda^{\prime}$. We put $B=0$ outside $\Lambda$. Then the following inequality holds

$$
L^{d-2} \sum_{c \in \Lambda^{\prime}}\left|\left(Q_{1} B\right)(c)\right|^{2}+\sum_{p}\left|\left(\partial_{1} B\right)(p)\right|^{2} \geqq \frac{1}{12 d^{2}} L^{-d-1}\|B\|^{2} .
$$


Another consequence is a bound from below for the form (2.120), or for the form (2.122). These forms are bounded from below by $\gamma_{0}^{\prime \prime}\|B\|^{2}$ with a positive constant $\gamma_{0}^{\prime \prime}$ dependent on $d$ and $L$ only. This implies that a covariance $\widetilde{C}_{\Lambda}^{(j)}$ of the Gaussian integral in (2.119) is bounded from above by a positive constant dependent on $d$ and $L$ only, and it has an exponential decay with a decay rate having the same property. We may calculate the integral in (2.119), and we get finally the desired identity:

$$
\begin{aligned}
\langle J, G J\rangle= & \left\langle J, \partial H_{j}^{\prime} C_{\Lambda}^{\prime(j)} H_{j}^{\prime *} \partial^{*} J\right\rangle \\
& +\left\langle J-\partial \Delta H_{j}^{\prime} C_{\Lambda}^{(j)} H_{j}^{* *} \partial^{*} J,\left(\tilde{G}_{j}+H_{j} \widetilde{C}_{\Lambda}^{(j)} H_{j}^{*}\right)\left(J-\partial \Delta H_{j}^{\prime} C_{\Lambda}^{(j)} H_{j}^{\prime *} \partial^{*} J\right)\right\rangle .
\end{aligned}
$$

We have to investigate yet the operators $\widetilde{G}_{j}, H_{j}$, and $H_{j}^{\prime}$. Doing similar calculations as in Sect. E. (1.91)-(1.103), in fact much simpler, we get the formula

$$
H_{j}=G_{j} Q_{j}^{*}\left(Q_{j} G_{j} Q_{j}^{*}\right)^{-1} .
$$

Thus this operator coincides with the operator introduced in Sect. D. For $\widetilde{G}_{j}$ we get

$$
\tilde{G}_{j}=G_{j}-G_{j} Q_{j}^{*}\left(Q_{j} G_{j} Q_{j}^{*}\right)^{-1} Q_{j} G_{j} .
$$

From these representations we obtain all the necessary properties of the operators $H_{j}, \widetilde{G}_{j}$. They follow from the Proposition 1.2 and from the formulas and the inequalities (1.99)-(1.101) for $Q_{j} G_{j} Q_{j}^{*}$. The operator $H_{j}^{\prime}$ can be investigated using the momentum representation obtained from (2.101)

$$
\widetilde{\left(H_{j}^{\prime} \mu\right)}\left(p^{\prime}+l\right)=\frac{\overline{u_{j}\left(p^{\prime}+l\right)}}{\Delta^{2}\left(p^{\prime}+l\right)}\left(\sum_{l^{\prime}} \frac{\left|u_{j}\left(p^{\prime}+l^{\prime}\right)\right|^{2}}{\Delta^{2}\left(p^{\prime}+l^{\prime}\right)}\right)^{-1} \tilde{\mu}\left(p^{\prime}\right) .
$$

This representation together with the analyticity method described in [3] imply that derivatives of $H_{j}^{\prime}$ up to third order, and their local Hölder norms as in (2.67), are uniformly bounded and have a uniform exponential decay with a decay rate depending on $d$ only.

All the above considerations imply the following

Proposition 2.5. The operator $G_{\square}$ defined by (2.90) on the torus $T_{\square}$ (or on the whole lattice $\left.\xi Z^{d}\right)$ has the representation (2.129) and satisfies all the inequalities (1.110)(1.114) of the Proposition 1.2 with a positive constant $\delta_{2}$ instead of $\delta_{0}$. This constant depends on $d$ and L only.

Let us make a remark connected with Proposition 2.5. If the set $B^{j}(\Lambda)$ equals $T_{\square}$ then the representation (2.129) relates the operator $G_{j+1}$ defined by averaging operations of the order $j+1$, to operators defined by the operations of the order $j$, and to unit lattice operators. Thus it is a recursive equation of the type studied in [3], for example the equation (2.34) in [3]. There these equations provided a basic method of proof of the regularity properties for the corresponding propagators. Unfortunately Eq. (2.129) relates $G_{j+1}$ to $\tilde{G}_{j}$ in a much more complicated way, the operator $\widetilde{G}_{j}$ is multiplied by the other operators. When we iterate the recursive equations we get long products of these operators and it is very hard to estimate such expressions. Of course this difficulty is connected with the fact that we have different gauge conditions on different scales. We circumvented the problem by 
reduction to Sect. F of paper [4], which ultimately relies on much simpler recursions.

Now let us come back to the equality (2.91). At first we will formulate the relevant inequalities for $G_{\square}$ rescaled back to $\eta$-scale. We have

$$
\left|\left(G_{\square} J\right)(x)\right|, \quad\left|\left(\nabla G_{\square} J\right)(x)\right| \leqq O(1)\left[\left(L^{j} \eta\right)^{2}, L^{j} \eta\right] e^{-\delta_{2}\left(L^{j} \eta\right)^{-1} \operatorname{dist}\left(\Delta, \Delta^{\prime}\right)}|J|,
$$

for $x \in \Delta(y), \operatorname{supp} J \subset \Delta\left(y^{\prime}\right), y, y^{\prime} \in \mathfrak{B} \cap T_{\square}$. Applying the inequalities (2.133), (2.88) and the remarks after the inequality (2.68) we obtain

$$
\left|\left(K_{\square, \square}, G_{\square}, h_{\square} J\right)(x)\right| \leqq O\left(M^{-1}\right) e^{-\frac{1}{2} \delta_{2} d\left(y, y^{\prime}\right)}|J|
$$

for $x \in \Delta(y), \operatorname{supp} J \subset \Delta\left(y^{\prime}\right)$, and this together with (2.91) implies

$$
|(R J)(x)| \leqq O\left(M^{-1}\right) e^{-\frac{1}{2} \delta_{2} d\left(y, y^{\prime}\right)}|J|, \quad x \in \Delta(y), \quad \operatorname{supp} J \subset \Delta\left(y^{\prime}\right) .
$$

Reasoning in the same way as in the proof of Proposition 2.2 we obtain

Proposition 2.6. There exists a positive constant $\delta_{3}$ depending on $d$ and L only, such that

$$
\begin{gathered}
|(G J)(x)|, \quad|(\nabla G J)(x)|, \quad\left|\left(G \nabla^{*} J\right)(x)\right|, \\
|(\Delta G J)(x)| \leqq O(1)\left[\left(L^{j} \eta\right)^{2}, L^{j} \eta, L^{j} \eta, 1\right] e^{-\delta_{3} d\left(y, y^{\prime}\right)}|J|
\end{gathered}
$$

for $x \in \Delta(y), y \in \Lambda_{j}$, supp $J \subset \Delta\left(y^{\prime}\right)$, with the constant $O(1)$ depending on d and L only; $\|\zeta \nabla G J\|_{\alpha}, \quad\left\|\zeta G \nabla^{*} J\right\|_{\alpha} \leqq O(1)\left(L^{j} \eta\right)^{1-\alpha}\left(\|\zeta\|_{\alpha}^{\xi}+|\zeta|\right) e^{-\delta_{3} d\left(y, y^{\prime}\right)}|J|, \quad \xi=L^{-j}$

for $0 \leqq \alpha<1, \zeta \in C_{0}^{\infty}(\tilde{\Delta}(y))$ (the cube $\tilde{\Delta}(y)$ for $y \in \Lambda_{j}$ is a sum of $2^{d}$ unit cubes on the $L^{-j}$-scale, having $y$ as a corner $), \operatorname{supp} J \subset \Delta\left(y^{\prime}\right)$, with the constant $O(1)$ depending on $d, L$ and $\alpha(O(1) \rightarrow \infty$ if $\alpha \rightarrow 1)$;

$$
\left|\left(\nabla G \nabla^{*} J\right)(x)\right| \leqq O(1) e^{-\delta_{3} d\left(y, y^{\prime}\right)}\left(\|J\|_{\varepsilon}^{\xi^{\prime}}+|J|\right)
$$

for $0<\varepsilon<1, x \in \Delta(y), \operatorname{supp} J \subset \tilde{\Delta}\left(y^{\prime}\right), y^{\prime} \in \Lambda_{j^{\prime}}, \xi^{\prime}=L^{-j^{\prime}}$, with the constant $O(1)$ depending on $d, L$, and $\varepsilon(O(1) \rightarrow \infty$ if $\varepsilon \rightarrow 0)$;

$$
\left\|\zeta \nabla G \nabla^{*} J\right\|_{\alpha} \leqq O(1)\left(L^{j} \eta\right)^{-\alpha}\left(\|\zeta\|_{\alpha}^{\xi}+|\zeta|\right) e^{-\delta_{3} d\left(y, y^{\prime}\right)}\left(\|J\|_{\alpha+\varepsilon}^{\xi^{\prime}}+|J|\right)
$$

for $0 \leqq \alpha<1, \varepsilon>0, \alpha+\varepsilon<1, \zeta \in C_{0}^{\infty}(\tilde{\Delta}(y)), \operatorname{supp} J \subset \tilde{\Delta}\left(y^{\prime}\right)$, with the constant $O(1)$ depending on $d, L, \alpha$, and $\varepsilon(O(1) \rightarrow \infty$ if $\alpha \rightarrow 1$ or $\varepsilon \rightarrow 0)$;

$$
\begin{gathered}
\|\zeta G J\|, \quad\|\zeta \nabla G J\|, \quad\left\|\zeta G \nabla^{*} J\right\|, \quad\left\|\zeta \nabla G \nabla^{*} J\right\|, \quad\|\zeta \nabla \nabla G J\|, \\
\left\|\zeta G \nabla^{*} \nabla^{*} J\right\| \leqq O(1)\left[\left(L^{j} \eta\right)^{2}, L^{j} \eta, L^{j} \eta, 1,1,1\right]|\zeta| e^{-\delta_{3} d\left(y, y^{\prime}\right)}\|J\|
\end{gathered}
$$

if $\operatorname{supp} \zeta \subset \Delta(y), y \in \Lambda_{j}$, supp $J \subset \Delta\left(y^{\prime}\right)$, with the constant $O(1)$ depending on $d$ and $L$.

The operator $G$ can be represented as

$$
\begin{aligned}
G= & G_{0}(I-R)^{-1}=\sum_{n=0}^{\infty} G_{0} R^{n}=\sum_{\omega=\left(\square_{0}, \ldots, \square_{2 n}\right)} h_{\square_{0}} G_{\square_{0}} h_{\square_{0}} \\
& \cdot K_{\square_{1}, \square_{2}} G_{\square_{2}} h_{\square_{2}} \cdot \ldots \cdot K_{\square_{2 n-1}, \square_{2 n}} G_{\square_{2 n}} h_{\square_{2 n}},
\end{aligned}
$$

and the series above is convergent in the norms appearing in the inequalities (2.136)(2.140). 
Let us remark that the symbol $\|\cdot\|_{\alpha}^{\xi}$ indicates the scale $\xi$ for which the Hölder norm is taken.

Let us also repeat once more that this theorem holds for the operators $G(\Omega)$ with Dirichlet boundary conditions on $\Omega^{c}, \Omega \supset \Omega_{1}$.

Finally let us consider the operator $Q G Q^{*}$ and its inverse. We consider these operators on the $L^{2}$-space defined by (2.69) with sites replaced by bonds. The operator $Q G Q^{*}$ is positive, hence the inverse is well defined and positive also. We want to prove similar bounds as for the operator $Q^{\prime} G^{2} Q^{\prime *}$ and we will follow rather closely the arguments given for it. From (2.136) we have

$$
\left|\left(Q G Q^{*}\right)\left(b, b^{\prime}\right)\right| \leqq O(1)\left(L^{j} \eta\right)^{2}\left(L^{j^{\prime}} \eta\right)^{-d} e^{-\delta_{3} d\left(b, b^{\prime}\right)}, \quad b \in \Lambda_{j}, \quad b^{\prime} \in \Lambda_{j^{\prime}} .
$$

Keeping the same notations as before we consider the operators

$$
C_{\square}=\left(\left(Q G_{\square} Q^{*}\right) \uparrow_{\square}\right)^{-1}, \quad C=\sum_{\square \in \mathscr{D}} h_{\square} C_{\square} h_{\square} .
$$

At first let us investigate bounds on $C_{\square}$. We assume that we have the same geometric situation as previously, so

$$
\begin{aligned}
\left\langle B,\left(Q G_{\square} Q^{*}\right) \Gamma_{\square} B\right\rangle & =\left(L^{\dot{j}} \eta\right)^{d+2}\left\langle B,\left.\left(Q G_{\square}^{\xi} Q^{*}\right)\right|_{\square} B\right\rangle \\
& =\left(L^{\dot{j}} \eta\right)^{d+2}\left\langle Q^{\prime *} B, Q_{j} G_{\square}^{\xi} Q_{j}^{*} Q^{\prime *} B\right\rangle,
\end{aligned}
$$

where $Q^{\prime \prime}$ is defined, as in (2.119), by the quadratic form in $B$ in the expression (2.112), and the last scalar product above is on the unit scale. We put $B$ equal to 0 outside $\square$, and we omit the superscripts $\xi$ and $\square$ in the sequel. The expression on the right-hand side in (2.144) is equal to $\left\langle Q^{*} B, G Q^{*} B\right\rangle$ and is defined by the integral in (2.95) with $\langle A, J\rangle$ replaced by $\left\langle A, Q^{*} B\right\rangle=\langle Q A, B\rangle$. This expression is gauge-invariant with respect to gauge transformations given by $\lambda$ satisfying $Q^{\prime} \lambda=0$. In the calculations between (2.95) and (2.129) we made such transformations only, and we may drop terms which appeared because of the noninvariance of $\langle A, J\rangle$. We obtain

$$
\begin{aligned}
\left\langle B, Q G Q^{*} B\right\rangle & =\left\langle B, Q \widetilde{G}_{j} Q^{*} B\right\rangle+\left\langle B, Q H_{j} \widetilde{C}_{A}^{(j)} H_{j}^{*} Q^{*} B\right\rangle \\
& =\left\langle Q^{\prime *} B, Q_{j} \widetilde{G}_{j} Q_{j}^{*} Q^{\prime *} B\right\rangle+\left\langle Q^{\prime *} B, Q_{j} H_{j} \widetilde{C}_{A}^{(j)} H_{j}^{*} Q_{j}^{*} Q^{\prime *} B\right\rangle .
\end{aligned}
$$

The first term on the right-hand side of the last equality is equal to 0 by the definition of $\widetilde{G}_{j}$. From the definition of $H_{j}$ we have $Q_{j} H_{j}=I$, hence

$$
\left\langle B, Q G Q^{*} B\right\rangle=\left\langle Q^{\prime *} B, \widetilde{C}_{\Lambda}^{(j)} Q^{\prime *} B\right\rangle=\left\langle B_{1}, \widetilde{C}_{A}^{(j)} B_{1}\right\rangle,
$$

where $B_{1}$ is equal to $Q^{\prime \prime *} B$ everywhere except the bonds of $\bigcup_{y \in A^{\prime}} A x(y)$ at which it is equal to 0 . The operator $\widetilde{C}_{A}^{(j)}$ is an inverse to the operator of the quadratic form (2.120), hence it is bounded from below by an inverse of an upper bound of this form. Taking into account that $\left\|B_{1}\right\|^{2}$ is bounded from below by const $\|B\|^{2}$, we get

$$
\left\langle B,\left(Q G_{\square} Q^{*}\right) \uparrow_{\square} B\right\rangle \geqq \gamma_{0}\|B\|^{2}
$$

with a positive constant $\gamma_{0}$ depending on $d$ and $L$ only. Of course we have also a similar bound from above and an exponential decay of a kernel of the operator in (2.147). This implies the same properties for $C_{\square}^{\xi}$ with the corresponding bounds 
and a decay rate $\delta_{4}$. For the operator $C_{\square}$ we get

$$
\left|C_{\square}\left(b, b^{\prime}\right)\right| \leqq O(1)\left(L^{j} \eta\right)^{-d-2} e^{-\delta_{4}\left(L^{j} \eta\right)^{-1} \mid b_{-}-b^{\prime}-1}, \quad b, b^{\prime} \in \mathfrak{B} \cap \square .
$$

We form the equality for $Q G Q^{*} C$ in exactly the same way as in (2.82) replacing only in the definition of $R_{\square, \square}$, the operators $Q^{\prime}, G^{\prime}(\tilde{\square})^{2}, G^{\prime 2}$ by $Q, G_{\square}, G$. We have the same estimates now as before, but with powers of scaling factors changed properly (we replace +4 and -4 in (2.83) by +2 and -2 ), thus we have (2.85) and this implies

Proposition 2.7. The operator $\left(Q G Q^{*}\right)^{-1}$ is given by the convergent expansions of the form (2.86), and it satisfies the bound

$$
\left|\left(Q G Q^{*}\right)^{-1}\left(b, b^{\prime}\right)\right| \leqq O(1)\left(L^{j} \eta\right)^{-2}\left(L^{\prime} \eta\right)^{-d} e^{-\frac{1}{2} \delta_{4} d\left(b, b^{\prime}\right)}, \quad b \in \Lambda_{j}, \quad b^{\prime} \in \Lambda_{j^{\prime}} .
$$

We will apply the results obtained until now to many different problems. At first let us consider the operator $H$. We have

Corollary 2.8. A kernel of the operator $H$,

satisfies the inequality

$$
(H B)(b)=\sum_{c \in \mathfrak{B}}\left(L^{j(c)} \eta\right)^{d} H(b, c) B(c),
$$

$$
\begin{gathered}
|H(b, c)|, \quad|(\nabla H)(b, c)|, \quad\|(\zeta \nabla H)(\cdot, c)\|_{\alpha} \\
\leqq O(1)\left[1,\left(L^{j} \eta\right)^{-1},\left(L^{j} \eta\right)^{-1-\alpha}\left(\|\zeta\|_{\alpha}^{\xi}+|\zeta|\right)\right]\left(L^{j^{\prime}} \eta\right)^{-d} e^{-\delta_{5} d\left(y, c_{-}\right)},
\end{gathered}
$$

$b \in \Delta(y)$ or $\operatorname{supp} \zeta \subset \Delta(y), y \in \Lambda_{j}, c_{-} \in \Lambda_{j^{\prime}}$.

This Corollary and Proposition 2.6 are our main technical results. They will be used systematically in subsequent papers.

Next let us consider the inequality (2.128) in Lemma 2.4 again. We will apply it in the following situation. Doing a $k+1$ renormalization transformation we have to calculate an integral of the form

$$
\text { const } \int d B \delta(Q B) \delta_{A x}(B) e^{-\frac{1}{2}\left\langle B, \Delta_{k} B\right\rangle} F(B)
$$

on the whole lattice $T^{(k)}$, or on a subset $\Lambda \subset T^{(k)}$. Using (2.118) and (2.128) we get

$$
\left\langle B, \Delta_{k} B\right\rangle \geqq \frac{\gamma_{0}}{12 d^{2}} L^{-d-1}\|B\|^{2}, \quad \text { or } \quad \Delta_{k} \geqq \frac{\gamma_{0}}{12 d^{2}} L^{-d-1}
$$

on the subspace of $B$ satisfying: $Q B=0, B\left(\Gamma_{y, x}\right)=0$ for $x \in B(y)$. Let us denote the covariance of the Gaussian integration in (2.152) by $C^{(k)}$, or by $C_{A}^{(k)}$, hence

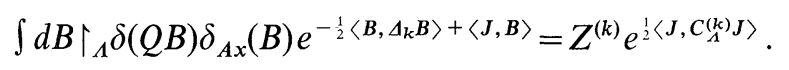

An easy way to get a useful representation for $C_{A}^{(k)}$ is to get rid of the unnecessary variables in the integral above. We remove the variables $B_{b}$ for $b \subset \Gamma_{y, x}$ using the $\delta$-functions $\delta_{A x}(B)$. Next we remove the variables $B_{b_{0}}$, where $b_{0}$ is a bond belonging to $B(c)$ for some $c \in \Lambda^{\prime}$, and contained in $c$, using the $\delta$-functions $\delta((Q B)(c))$. If we denote the remaining variables by $B^{\prime}$, then we can write $B=C B^{\prime}$, where $C$ is a linear 
operator, and we have

(the left-hand side of (2.154))

$$
\begin{aligned}
& =\left(L^{d}\right)^{\left|A^{\prime}\right|} \int d B^{\prime} e^{-\frac{1}{2}\left\langle B^{\prime}, C^{*} \Delta_{k} C B^{\prime}\right\rangle+\left\langle C^{*} J, B^{\prime}\right\rangle} \\
& =\left(L^{d}\right)^{\left|\Lambda^{\prime}\right|} Z^{\prime(k)} e^{\frac{1}{2}\left\langle C^{*} J,\left(C^{*} \Delta_{k} C\right)^{-1} C^{*} J\right\rangle},
\end{aligned}
$$

hence

$$
C_{\Lambda}^{(k)}=C\left(C^{*} \Delta_{k} C\right)^{-1} C^{*}
$$

By the definition of $C$ we have of course that $C B^{\prime}=0$ outside $\Lambda, Q C B^{\prime}=0$, $\left(C B^{\prime}\right)\left(\Gamma_{y, x}\right)=0, x \in B(y), y \in \Lambda^{\prime}$, for arbitrary $B^{\prime}$. The inequality (2.153) implies

$$
\left\langle B^{\prime}, C^{*} \Delta_{k} C B^{\prime}\right\rangle \geqq \frac{\gamma_{0}}{12 d^{2}} L^{-d-1}\left\|C B^{\prime}\right\|^{2} \geqq \gamma_{0}^{\prime}\left\|B^{\prime}\right\|^{2},
$$

where $\gamma_{0}^{\prime}=\frac{\gamma_{0}}{12 d^{2}} L^{-d-1} . C$ is a short-ranged operator, so $C^{*} \Delta_{k} C$ has the same exponential decay as $\Delta_{k}$. Now we may apply the theory developed in Sect. 5 of [3] on unit lattice operators. It gives us an exponential decay, and all the other properties, for the operator $\left(C^{*} \Delta_{k} C\right)^{-1}$, hence for $C_{\Lambda}^{(k)}$ also.

Such a scheme will be applied in the future to investigate all unit lattice propagators defined by generalizations of the integrals (2.152), (2.154).

\section{References}

1. Bałaban, T.: (Higgs) $)_{2,3}$ quantum fields in a finite volume. I. A lower bound. Commun. Math. Phys. 85, 603-636 (1982)

2. Bałaban, T.:(Higgs $)_{2,3}$ quantum fields in a finite volume. II. An upper bound. Commun. Math. Phys. 86, 555-594 (1982)

3. Bałaban, T.: Regularity and decay of lattice Green's functions. Commun. Math. Phys. 89, 571597 (1983)

4. Bałaban, T.: Propagators and renormalization transformations for lattice gauge theories. I. Commun. Math. Phys. 95, 17-40 (1984)

5. Brydges, D.C., Federbush, P.: A lower bound for the mass of a random Gaussian lattice. Commun. Math. Phys. 62, 79-82 (1978)

6. Brydges, D.C., Fröhlich, J., Seiler, E.: On the construction of quantized gauge fields. I. General results. Ann. Phys. 121, 227-284 (1979)

7. Wilson, K.G., Bell, T.L.: Finite-lattice approximations to renormalization groups. Phys. Rev. B 11, 3431-3445 (1975)

Communicated by A. Jaffe

Received January 12, 1984; in revised form June 28, 1984 